\title{
1 Highly task-specific and distributed 2 neural connectivity in working memory 3 revealed by single-trial decoding in 4 mice and humans
}

5 Daniel Strahnen ${ }^{1}$, Sampath K.T. Kapanaiah, Alexei M. Bygrave², Birgit Liss ${ }^{1,3}$, David M.

6 Bannerman ${ }^{4}$, Thomas Akam4, Benjamin F. Grewe ${ }^{5}$, Elizabeth L. Johnson ${ }^{6,7}$, Dennis Kätzel ${ }^{1, *}$

$8{ }^{1}$ Institute of Applied Physiology, Ulm University, Ulm, Germany

$9 \quad{ }^{2}$ Department of Neuroscience, Johns Hopkins University, USA

10 '3inacre College and New College, University of Oxford, Oxford, UK

$11{ }^{4}$ Department of Experimental Psychology, University of Oxford, Oxford, UK

$12{ }^{5}$ Institute of Neuroinformatics, ETH Zürich, Zürich, Switzerland

$13{ }^{6}$ Helen Wills Neuroscience Institute, University of California, Berkeley, Berkeley, CA, USA

$14{ }^{7}$ Life-Span Cognitive Neuroscience Program, Institute of Gerontology, Wayne State 15 University, Detroit, MI, USA

* Correspondence: dennis.kaetzel@uni-ulm.de; +49 731500 33770; Fax +49731500 33779; Institute of Applied Physiology, Ulm University, Albert-Einstein-Allee 11, 89081 Ulm,

Germany

Words: 5580 (excluding Abstract, Methods, References, Figure legends)

Figures: 8; Supplementary Figures: 14 


\section{Abstract}

23 Working memory (WM), the capacity to briefly and intentionally maintain mental items, is key to successful goal-directed behaviour and impaired in a range of psychiatric disorders. To date, several brain regions, connections, and types of neural activity have been correlatively associated with WM performance. However, no unifying framework to integrate these findings exits, as the degree of their species- and task-specificity remains unclear. Here, we investigate WM correlates in three task paradigms each in mice and humans, with simultaneous multi-site electrophysiological recordings. We developed a machine learningbased approach to decode WM-mediated choices in individual trials across subjects from hundreds of electrophysiological measures of neural connectivity with up to $90 \%$ prediction accuracy. Relying on predictive power as indicator of correlates of psychological functions, we unveiled a large number of task phase-specific WM-related connectivity from analysis of predictor weights in an unbiased manner. Only a few common connectivity patterns emerged across tasks. In rodents, these were thalamus-prefrontal cortex delta- and beta-frequency connectivity during memory encoding and maintenance, respectively, and hippocampalprefrontal delta- and theta-range coupling during retrieval, in rodents. In humans, taskindependent WM correlates were exclusively in the gamma-band. Mostly, however, the predictive activity patterns were unexpectedly specific to each task and always widely distributed across brain regions. Our results suggest that individual tasks cannot be used to uncover generic physiological correlates of the psychological construct termed WM and call for a new conceptualization of this cognitive domain in translational psychiatry. 


\section{Introduction}

45 Working memory (WM) refers to the capacity to store and manipulate contents of perception and thought at the forefront of attention over seconds to minutes ${ }^{1,2}$. Several psychiatric and neurological disorders are characterised by severe and pharmaco-resistant impairments of $\mathrm{WM}^{3}$. Studies in humans, non-human primates, and rodents ${ }^{4}$ have centrally implicated the prefrontal cortex $(\mathrm{PFC})^{5-12}$, parietal cortex ${ }^{11,13}$, medio-dorsal thalamus $(\mathrm{MD})^{14,15}$, and hippocampus ${ }^{8,16-21}$ in WM processes. In particular, connectivity between these brain structures correlates with the performance in specific tasks ${ }^{11,22-24}$ (see Supplementary Table 1 for an overview of rodent studies).

However, it remains elusive which kinds of neural connectivity actually mediate WM. Several - partially conflicting - reports suggest that specific brain regions, frequency bands ${ }^{25-27}$ and types of inter-regional coupling (or metrics to analyse) are essential for WM. Studies in rodents, for example, have implicated network oscillations in either the delta $(\delta)^{28}$, theta $(\theta)^{27,29,30}$, beta $(\beta)^{14,26}$, or gamma $(\gamma)^{25}$ frequency, in coupling between such frequency bands (esp. $\theta-\gamma)^{31}$ or between oscillations and local spiking of neurons ${ }^{14,27,31-33}$, or in interactions between either the ventral $(\mathrm{vHC})^{31,33}$ or dorsal hippocampus $(\mathrm{dHC})^{26,29,34}$ and PFC. On a Tmaze rewarded-alternation task, both $\mathrm{vHC} \rightarrow \mathrm{PFC}$ connectivity ${ }^{33}$ and $\mathrm{dHC}-\mathrm{PFC}$ coupling via the thalamic nucleus reuniens ${ }^{34}$ have been claimed to mediate the encoding of WM-contents using optogenetic inhibition experiments. In addition to this diversity of findings in rodents which mainly originate from recordings in spatial alternation tasks (Supplementary Table 1) it remains largely unknown to what extent the discovered WM substrates generalize across different tasks and species.

Moreover, it remains unclear if this diversity of reported associations between specific neural activities and WM performance implicates that, indeed, several types of connectivity and connections are engaged in WM simultaneously. Alternatively, it could be due to differences between task paradigms, species, specific rodent models, or analysis procedures. Several factors may explain the current uncertainty regarding the physiological correlates of WM. 
71 This includes the difficulty of separating correlates of more basic functions like motivation, spatial processing, and attention from the actual WM component of the task - especially in rewarded-alternation assays that are mostly used in rodents (Supplementary Table 1) ${ }^{35}$.

74 Further uncertainty arises from the typical analytical approach of correlating within-subject averages of performance (WM accuracy score) $)^{26,29,31}$, trial type (correct vs. incorrect) ${ }^{33}$, or trial phase (sample vs. choice phase) ${ }^{30,33}$ with within-subject averages of a select connectivity measure. Such correlations do not necessitate mechanistic causation but could be indirect or even epiphenomenal. Finally, the streetlight effect has recently been highlighted as a rather principal limitation of studies of physiological correlates of psychological function ${ }^{36}$. In this context, this term denotes the missing of neurophysiological correlates due to a biased selection of only few investigated brain regions, connections, or activity parameters. We recently showed that the rich plethora of widely used functional connectivity measures indeed contains a considerable number of non-redundant metrics ${ }^{37}$, which entails the possibility for seemingly contradictory findings and inconclusive null results.

To identify inter-regional neural connectivity that is associated with WM in mice and humans, we addressed these issues in several ways: First, we comparatively assessed three distinct visual WM tasks in each species. In mice, we applied an operant 5-choice delayedmatching-to-sample (DMTS) task as well as a T-maze-based and an operant 2-choice delayed-non-matching-to-sample (DNMTS) task. In humans, we analysed an existing dataset where three distinct task types which featured the same temporal structure and were inter-mixed within one session ${ }^{20}$. Therefore, in each species, the non-WM related differences between the applied tasks were relatively small, which benefits an optimal comparison of the neurophysiological basis of different types of WM. Second, during these tasks, local field potentials (LFPs) were simultaneously recorded from four sites (PFC, dHC, vHC, and MD thalamus) in rodents, and from three sites (PFC, medial temporal lobe, MTL, and orbitofrontal cortex, OFC) in humans. Third, to maximize connectivity-related information 
98

99

100

101

102

103

104

105

106

107

108

109

110

111

112

113

114

115

116

117

118

regional neural coupling. The composition of this set was optimized based on our prior analysis of redundancies between the most commonly used LFP-based coupling metrics ${ }^{37}$. Each metric was determined in three or four task phases, four or five frequency bands, and as both absolute and relative measures. This yielded a total of 960 and 1344 connectivity measures in mice and humans, respectively, in addition to $\sim 240$ measures of local activity. Finally and most importantly, we applied machine learning (ML) to predict WM choices on a single-trial basis from these high-dimensional patterns of functional connectivity. Data-driven approaches have recently been deployed to reduce the streetlight effect ${ }^{36,38-40}$. These studies have applied unsupervised classification procedures to extract electrophysiological correlates of stress responses ${ }^{39}$, depression vulnerability ${ }^{38}$, and anxiety ${ }^{36}$ from connectivity patterns obtained from multi-site LFP-recordings in mice, collectively termed the electome. To establish a largely unbiased search for WM correlates within high-dimensional electome patterns, in contrast, we translated the supervised, trial-based nature of WM tasks (predefining choices as correct or incorrect) into our analysis ${ }^{10,21,24,33,41,42}$. Implementing a supervised decoding analysis allowed us to harness the power of prediction to prove the presence of behaviourally relevant information in complex patterns of neural signals ${ }^{43}$. The possibility of trial-by-trial prediction of WM-based choices from the spiking activity of large ensembles of neurons in rodent PFC ${ }^{10,33}$, monkey inferior temporal cortex ${ }^{41}$, or human hippocampus ${ }^{21}$ has recently been demonstrated. In these attempts, however, prediction was done with classifiers that were trained specifically for a single animal and task session, since the predictor variables were individual neurons. In contrast, we applied ML across datasets from multiple subjects and sessions and used connectivity and activity metrics from multiple sites as predictor variables. In this way, subsequent quantification of the predictive power of each electrophysiological variable allowed a largely unbiased identification of connectivity patterns associated with WM in mice and humans as verified by trial-by-trial predictive power. 
Correct choices in DMTS working memory are associated with distinct signatures of connectivity

To evaluate associations between WM and electrophysiological measures of inter-regional connectivity, we first implanted mice with chronic field electrodes in PFC, MD, vHC, and dHC and tested them in three spatial working memory (SWM) tasks (Fig. 1a-f): first, an operant DMTS 5-choice SWM (5-CSWM) task, subsequently the T-maze rewarded alternation task, and finally an operant DNMTS 2-choice SWM (2-CSWM) task. To allow for simultaneous LFP recordings during operant tasks, we developed a custom-designed operant box optimized for implanted and tethered animals (Fig. 1c) that is tightly integrated with electrophysiological recordings via pyControl software and microcontroller modules ${ }^{44}$. The set of three tasks (Fig. 1d-f) was chosen to retain comparability due to their shared visuospatial nature and distinct individual differences (i.e., operant DMTS vs. DNMTS; mazebased DNMTS vs. operant DNMTS). In addition, the T-maze task was included due to its wide usage (see e.g., Supplementary Table 1). The 5-CSWM was specifically designed to limit the usage non-WM mediation strategies by the animal - due the large number of choice configurations, requirement to shuttle between opposite walls of the box, and delay-periods in total darkness, as we described in detail recently ${ }^{45}$. Also, both operant tasks provide tight control over the timing of behavioural events and deliver intrinsic control variables for WMenabling psychological functions like attention, measured by sample phase (SP) accuracy, and motivational drive, measured by reward latency. In each task, we applied extensions of

146 the delay between the SP and the choice phase (CP) across which the memorized

147 information needed to be maintained in order to strongly engage WM capacity. As expected 148 for WM assays ${ }^{2}$, such delay challenges significantly decreased WM choice accuracy of the 149 mice (Fig. 1g-i). 
a

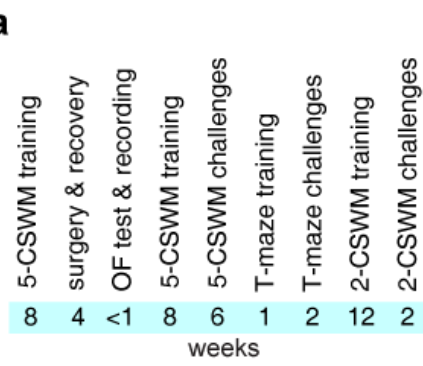

d Operant DMTS (5-CSWM)

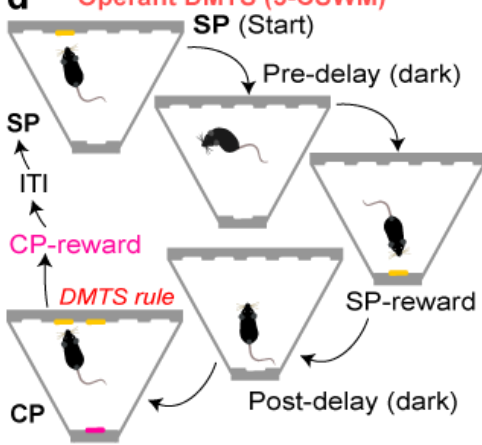

g

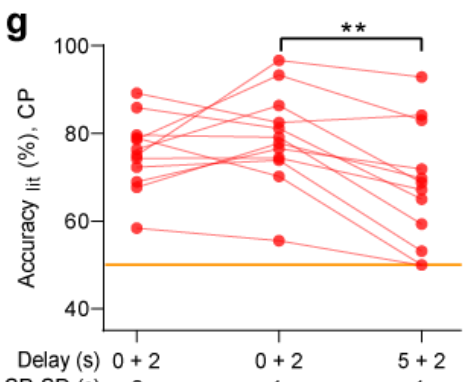

b

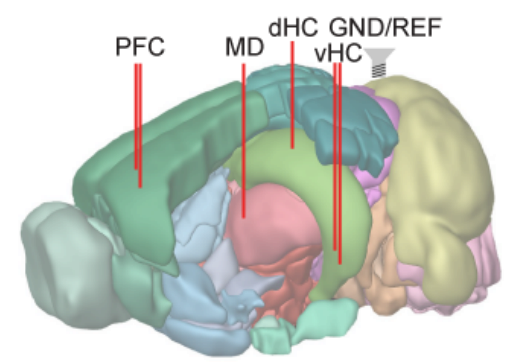

e

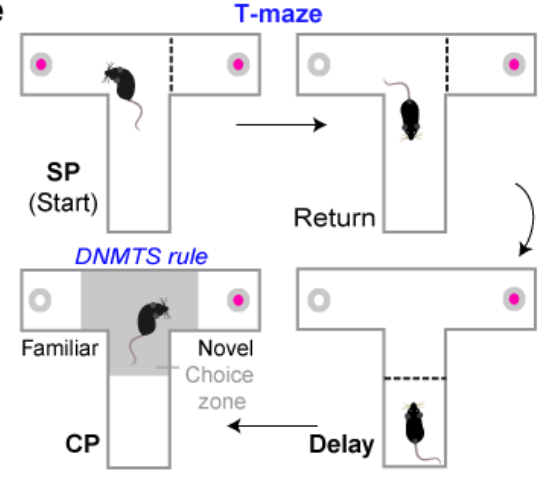

h

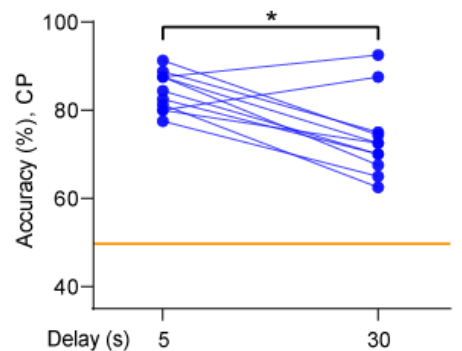

C

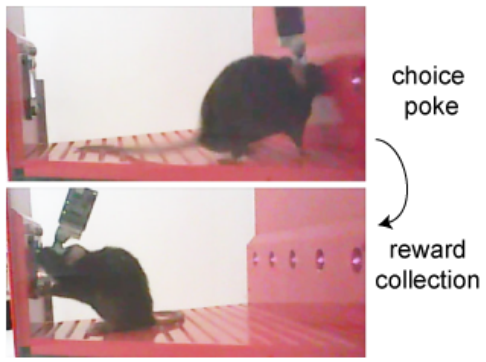

$\mathbf{f}$
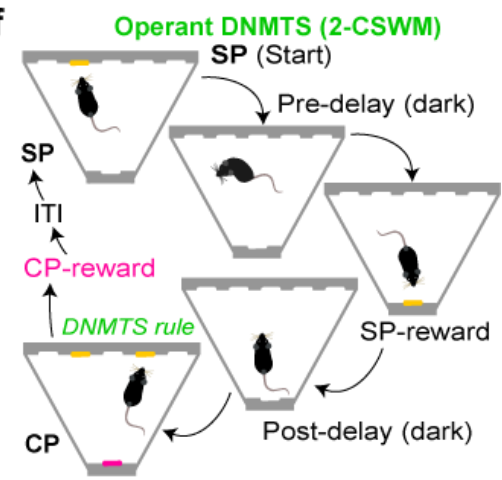

i

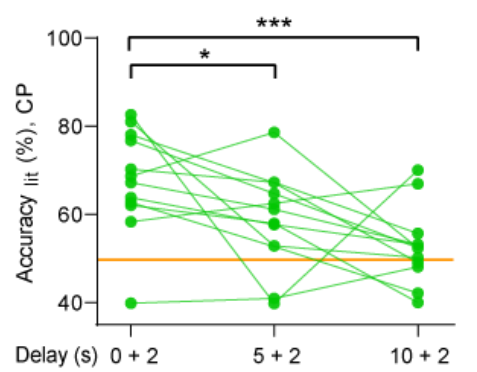

Fig. 1 | Rodent SWM tasks. (a) Timeline of experiments in the analysed cohort (see

Methods). (b) Illustration of electrode placements in the mouse brain (image taken from

Allen Brain Atlas); pairs of electrodes were inserted into PFC and vHC. (c) Lack of obstruction of tethered mice with mounted headstage during poking of choice hole (top) and reward collection (bottom) in custom-made pyControl operant boxes. (d-f) Illustration of DMTS 5-CSWM ${ }^{45}$ (d), T-maze rewarded alternation (e), and DNMTS 2-CSWM (f) tasks; in $(d, f)$, choices in SP and CP need to be made at the 5-choice wall (top), while rewards for correct responses in each phase are collected on the opposite wall (magenta, bottom). (e-i) WM performance measured as response accuracy in the CP (\% correct choices relative to available indicated options) in 12 mice in each set of challenge conditions including their respective baseline with simultaneous electrophysiological recordings. Delay length determining the WM challenge stated on x-axes; for operant tasks $(e, j)$ pre- + post-delay 
(referring to set delays before and after SP-reward collection) are indicated. SP-SD, stimulus duration in sample phase. Asterisks indicate differences between challenge, Sidak-post-hoc tests conducted after significant main effect of challenge, RM-ANOVA. Orange line, chance level performance. ${ }^{*} P<0.05 ;{ }^{* *} P<0.01 ;{ }^{* *} P \leq 0.001$.

170 To initially explore possible WM correlates among measures of neural connectivity, we computed time-resolved spectrograms of four largely non-redundant ${ }^{37}$ connectivity metrics aligned to the time of correct and incorrect SP and CP responses for the distinct phases of

173 the 5-CSWM task (non-directed: coherence, Coh; weighted Phase-Lag-index, wPLI;

174 directed: Granger causality, GC; partial directed coherence, PDC; Supplementary Fig. 1-2). We subtracted spectra of correct SP responses or incorrect CP responses from those of correct $\mathrm{CP}$ responses to eliminate neural representations of poking action, execution of a reward-related response, and attention (Fig. 2a-d, Supplementary Fig. 3). This qualitative analysis suggested a complex pattern of connectivity associated with correct WM decisions,

179 including elevated hippocampal-prefrontal connectivity in the low $\gamma$-range $(30-48 \mathrm{~Hz})$

180 immediately after the CP response (Fig. 2a-b) and sustained connectivity in the PFC $\rightarrow$ MD and $\mathrm{vHC} \rightarrow \mathrm{dHC} \theta$-range $(5-12 \mathrm{~Hz})$ during the delay (Fig. 2c-d). 
bioRxiv preprint doi: https://doi org/10.1101/2021.04 20.440621; this version posted April 21, 2021. The copyright holder for this preprint (which was not certified by peer review) is the author/funder, who has granted bioRxiv a license to display the preprint in perpetuity. It is made available under aCC-BY 4.0 International license.
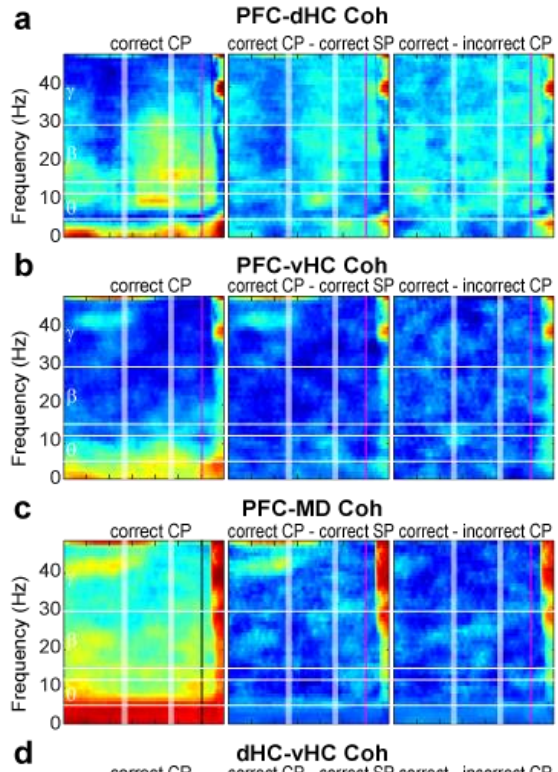

d $\quad$ dHC-vHC Coh
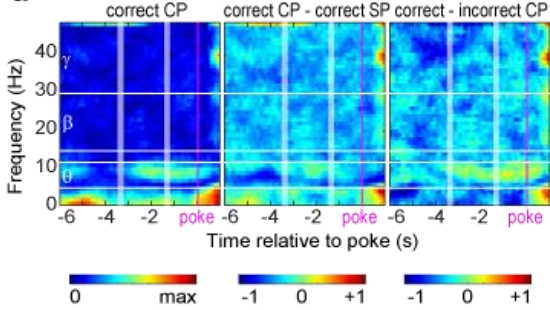

$\mathrm{PFC} \rightarrow \mathrm{dHC}$ GC

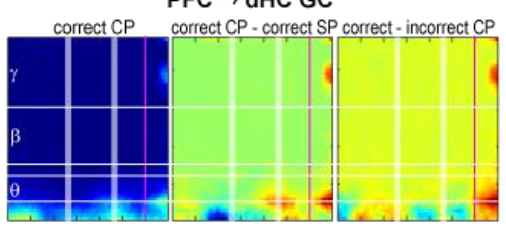

$\mathrm{PFC} \rightarrow \mathrm{vHC}$ GC

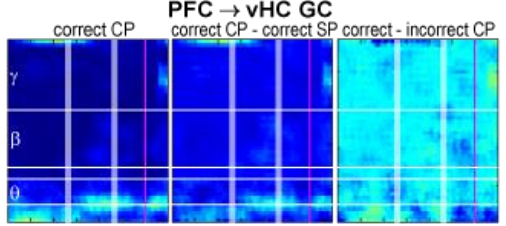

$\mathrm{PFC} \rightarrow \mathrm{MD}$ GC

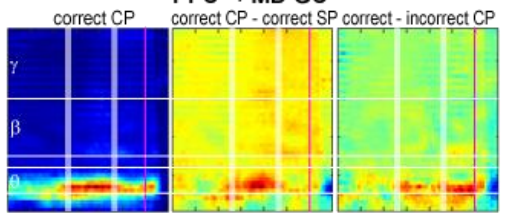

$\mathrm{dHC} \rightarrow \mathrm{vHC}$ GC

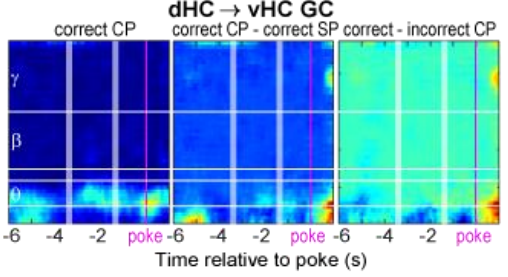

Time relative to poke (s)

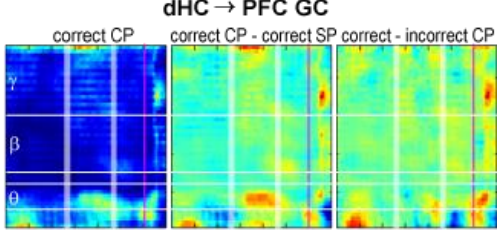

$\mathrm{vHC} \rightarrow$ PFC GC

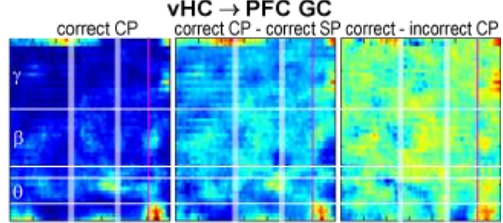

$\mathrm{MD} \rightarrow$ PFC GC

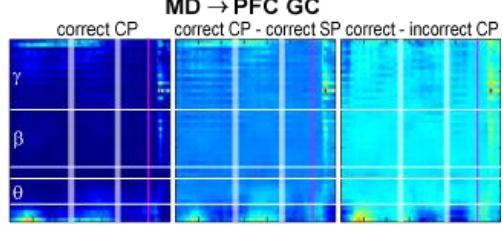

$\mathrm{vHC} \rightarrow \mathrm{dHC}$ GC

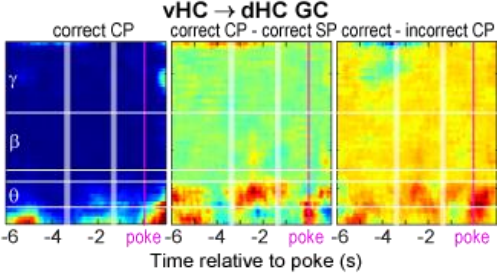

Time relative to poke (s)

Fig. 2 | Connectivity in the DMTS 5-CSWM task. (a-d) Spectrograms depicting min-max

normalized coherence (Coh) and Granger causality (GC) for the connections stated above each triplet panel for the delay and CP of the 5-CSWM task, temporally aligned to the choice poke entry ( $p$, vertical magenta lines) showing $6 \mathrm{~s}$ before until $1 \mathrm{~s}$ after the choice poke ( $\mathrm{x}$ axes in $(d))$; the start and end of the post-delay shown by white stripes corresponding to mean $\pm S D$ as determined by CP response latency. Each triplet shows the absolute value (left), the difference between the former and either the prior correct SP (middle), or incorrect CPs (right). Horizontal white lines show borders between analysed frequency bands, stated on the left. See Supplementary Fig. 1-2 for spectrograms of absolute values in correct and display as (a-d) for wPLI and PDC. 
Trial-by-trial prediction of DMTS WM-mediated choices from local and longrange neural activity

197 The ability to predict behavioural choices from neural activity may be regarded as evidence that such activity encodes aspects of these choices ${ }^{33,43}$. We assessed individual connectivity variables as five separate connectivity metrics (Coh, wPLI, GC, PDC, and cross-regional $\theta-\gamma$ phase-amplitude coupling, PAC) in four task phases (SP, pre-reward delay, post-reward delay, CP; see Fig. 1d), and four frequency bands $(\delta, 1-4 \mathrm{~Hz}, \theta, 5-12 \mathrm{~Hz}, \beta, 15-30 \mathrm{~Hz}$, low- $\gamma$, 30-48 Hz) along 4 connections (as shown in Fig. 2a-d). In the same way, indicators of local activity (power and local $\theta-\gamma \mathrm{PAC}$ ) were calculated within the four involved regions (dHC, vHC, PFC, MD). Additionally, all metrics were calculated in relative terms by dividing their obtained value by the value of that specific metric during the inter-trial-interval (ITI) before the start of the respective trial. This resulted in 240 variables for each inter-regional connection and 56 intra-regional activity variables for each area characterizing every 5CSWM trial. We trained subspace discriminant classifiers - which proved superior among 25 different types of linear and non-linear classifiers (Supplementary Fig. 4) - to predict WM-

210 choice trial-by-trial using the parameters contributed by each connection or region separately 211 for trials of the final 5-CSWM challenge ( $1 \mathrm{~s} \mathrm{SP}$ stimulus duration, SP-SD, $5 \mathrm{~s}$ delay; Fig. 212 3a). A single decoding model was generated across all subjects and its decoding accuracy 213 was determined by predicting trials that were not part of the training dataset, i.e. by cross214 validation.

215 We found that individual 5-CSWM choices could be decoded with $79.4 \%$ and $79.8 \%$ 216 maximum average accuracy when using measures of neural connectivity along the PFC-MD 217 or the PFC-dHC connection as predictors, respectively (Fig. 3b). Using one-way ANOVA and 218 pairwise Tukey post-hoc tests, we established a hierarchy between decoding accuracies 219 obtained from each of the four connections and four regions. Decoding accuracies obtained 220 from PFC-MD, PFC-dHC, local dHC and MD activity did not differ from each other and were 221 superior to the remainder (Fig. 3b). Even though decoding accuracies varied by connection 
bioRxiv preprint doi: https://doi.org/10.1101/2021.04 20.440621; this version posted April 21, 2021. The copyright holder for this preprint (which was not certified by peer review) is the author/funder, who has granted bioRxiv a license to display the preprint in perpetuity. It is made available under aCC-BY 4.0 International license.

and region, they were always significantly better than those of control classifiers trained with shuffled labels $\left(P<10^{-17}, t\right.$-tests) which, in turn, decoded indistinguishable from the $50 \%$ chance level on average (Fig. 3b). To evaluate the generality of the obtained classifiers, we assessed if they could also decode WM-based choices in data from other 5-CSWM challenge protocols. Even though the resulting decoding accuracies were generally lower compared to those achieved with data from the same protocol, they were still significantly higher than those of classifiers trained with shuffled labels (Supplementary Fig. 5). These analyses reveal that WM-based choice is encoded in LFP-based connectivity and activity measures in individual trials and that such information is widely distributed across multiple brain regions.
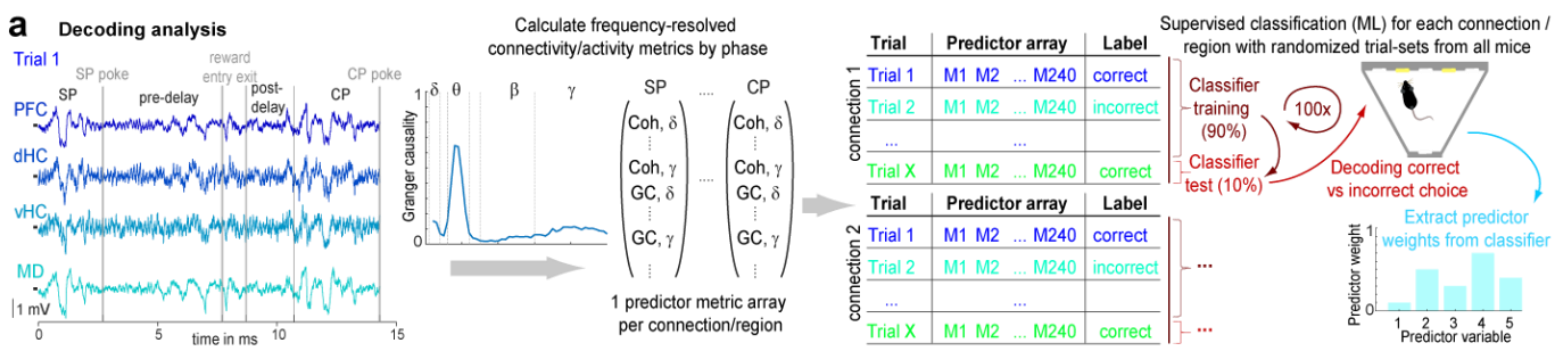

b

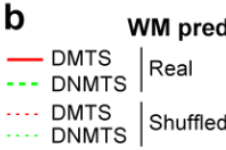
$\cdots .$. DNMTS
$\cdots$ DMTS
$\cdots$ DNMTS (3) 4

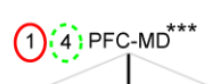
C WM prediction accuracy (\%), T-maze $\begin{aligned} & \text { - T-maze, } 5 \mathrm{~s} \\ & \cdots \text {-.- T-maze, } 30 \mathrm{~s}\end{aligned} \mid$ Real … T-maze, 5s $\mid$ T-maze, 30s $\mid$ Shuffled

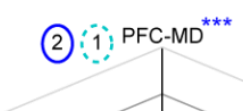

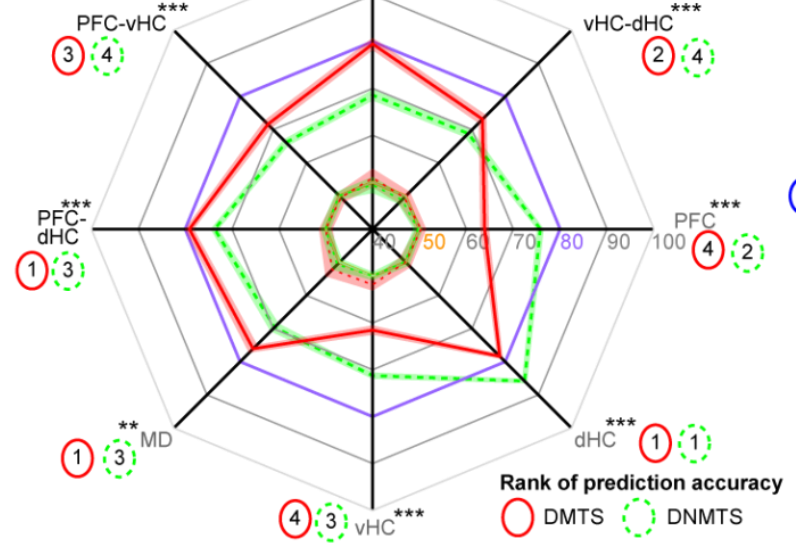
(3)
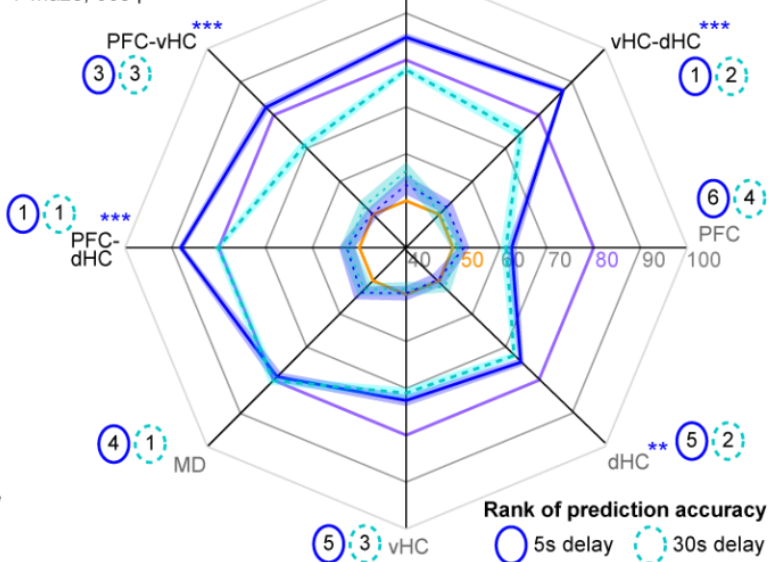
analysis (see Methods). (b-c) Cross-subject decoding accuracies achieved on average when using connectivity or local activity parameters of the indicated individual connections (black) or areas (grey), respectively to predict WM-based correct vs. incorrect choices in the DMTS 
5-CSWM task (combined $1 \mathrm{~s}$ SP-SD, 5+2 s delay challenge, 2 sessions; red, b), the DNMTS

2-CSWM task (baseline, 2 sessions, green, b), the T-maze WM task with either 5s (solid

blue, 4 sessions, c) or 30s (dashed blue, 4 sessions, c). Thinner dotted lines show decoding accuracies of corresponding classifiers trained with shuffled labels, remaining at chance level (50\%, orange). Classifiers trained with real labels perform better than those trained with shuffled labels in all cases $\left(P<10^{-17}, t\right.$-tests, not indicated). The accuracy of $80 \%$ is coloured in purple to aid comparison. Numbers in coloured ovals indicate the rank of the prediction accuracies achieved on average by using data from the respective connection or region.

Ranks have been generated from pairwise comparisons with Tukey post-doc tests

all cases); connections/regions that were not significantly different from each other were assigned the same rank. Black stars in (b) indicate differences of accuracy values achieved in the two operant tasks (Tukey post-hoc tests after significant effect of task-type in ANOVA across all three tasks). Blue stars in (c) indicate pairwise differences between the two delays (uncorrected $t$-tests). ${ }^{* *} P<0.01 ;{ }^{* \star *} P \leq 0.001$. Shaded regions around mean show s.e.m. across 100 classifiers generated for each task and connection or region.

Specific connections and regions are engaged differently in distinct rodent

\section{WM tasks}

255 To investigate if this conclusion applies generally to rodent WM, we repeated the same analysis for the operant DNMTS data (final baseline sessions, $2 \mathrm{~s}$ delay). In this case, however, we obtained the maximum average prediction accuracy $(86.1 \%)$ from local $\mathrm{dHC}$ activity, rather than PFC-MD (66.1\%, lowest rank of all classifiers) or PFC-dHC (73.5\%) connections. Generally, in this task, local activities allowed relatively high decoding accuracies $(72-77 \%$ for PFC, MD, and $\mathrm{vHC})$, while coupling metrics were significantly less predictive (66-68\%, $P<0.001$, Tukey; except for dHC-PFC, Fig. 3b). 
connections being most predictive), whereas local activities were significantly less predictive

(62-79\%; $P<0.001$, Tukey, Fig. 3c). However, decoding accuracies for information from all

4 connections decreased when analysing data from the $30 \mathrm{~s}$ delay challenge, in which these animals also showed lower behavioural performance (Fig. 1h, 3c), suggesting that not only task type but also task difficulty affect the information encoded in each connection.

Specific connections and regions are engaged differently in distinct phases of the rodent DMTS 5-CSWM task

271 The prior analyses entail at least two conclusions: Firstly, WM-related information in a single 272 trial is not encoded in any single region or connection, although some of them bear higher 273 predictive power regarding WM-choice than others. Secondly, the predictive power of a 274 given region or connection is not uniform but strongly depends on the type of WM task, 275 indicating that different mechanisms and regions are engaged to solve distinct behavioural demands. These conclusions re-emphasize the question as to what extent oscillatory 277 processes in distinct frequency bands, of a distinct biological type, or in a specific task phase 278 (encoding, delay, choice) can be regarded as correlates of WM (Supplementary Table 1).

279 To answer this question, we took advantage of the fact that a linear classifier reveals the 280 predictive power of each involved predictor variable according to its assigned weight. We performed Bonferroni-adjusted $t$-tests comparing the weights for each connectivity variable with the weights assigned by the classifiers trained on label-shuffled control data, and, additionally, conducted $t$-tests comparing the amplitudes of each variable between correct and incorrect trials that contributed to the classifiers. Variables for which both $t$-tests were significant were considered as bearing WM-related information (indicated by colour in Fig. 4a). This analysis revealed a relatively small set of consistent WM-related feature-classes as correlates of DMTS 5-CSWM, a majority of them in the $\gamma$-range (Fig. 4b): (1) PFC $\rightarrow$ MD $\delta$ and $\gamma$-range connectivity in the $S P$, (2) MD $\rightarrow$ PFC $\beta$ - and $\gamma$-range as well as $\mathrm{dHC}$-vHC 
bioRxiv preprint doi: https://doi.org/10.1101/2021.04.20.440621; this version posted April 21, 2021. The copyright holder for this preprint (which was not certified by peer review) is the author/funder, who has granted bioRxiv a license to display the preprint in perpetuity. It is made available under aCC-BY 4.0 International license.

a

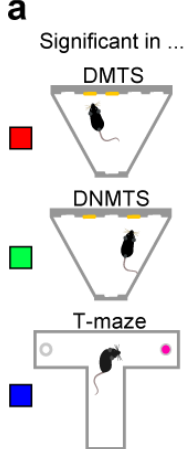

$\triangle \mathrm{DMTS}+$ DNMTS

$\square \mathrm{DMTS}+\mathrm{T}$-maze

$\square$ DNMTS + T-maze

All 3 combined

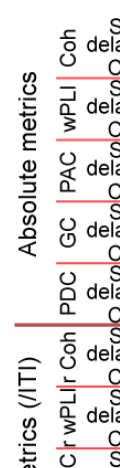

SP

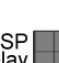
SP
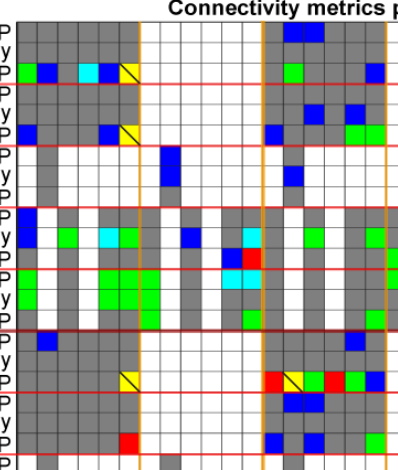

ar

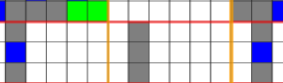

10

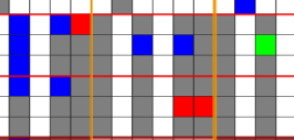

$\sqrt{x+1}$
Connectivity metrics prediciting WM in all three tasks (WT mice)

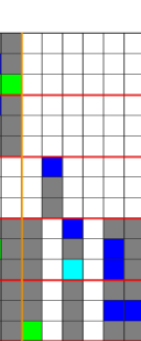

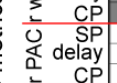

$\begin{array}{ll} & \\ 0 & S P \\ 0 & \text { delay } \\ 0 & C \\ 0 & \end{array}$

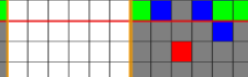
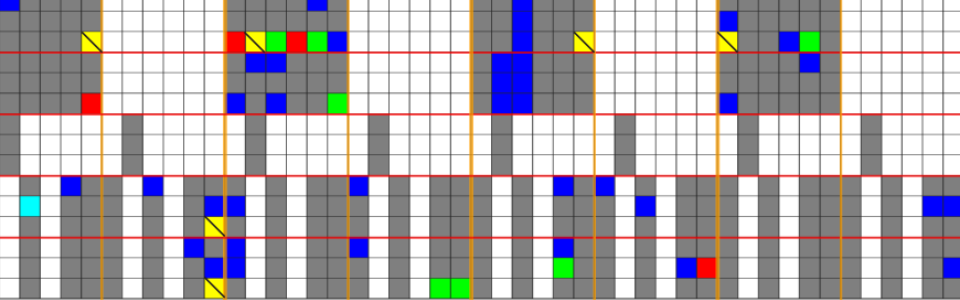

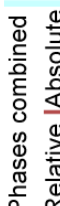

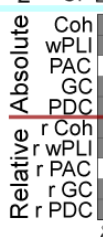

Coh
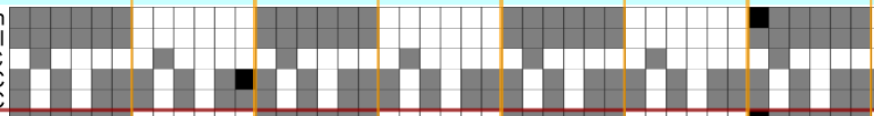

PAI

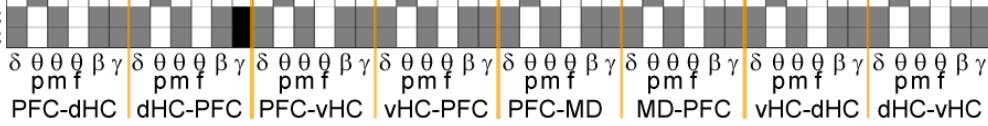

C DMTS - absolute metric predictors

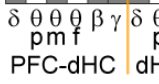

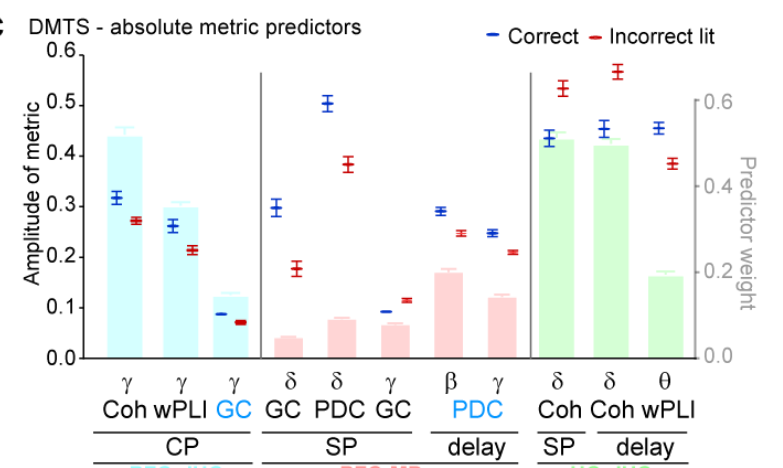

d DMTS - relative metric predictors

b DMTS

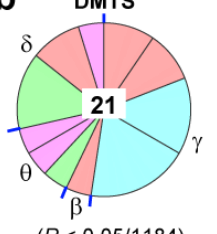

$(P<0.05 / 1184)$

DNMTS

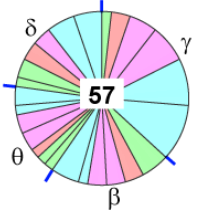

$(P<0.05 / 888)$

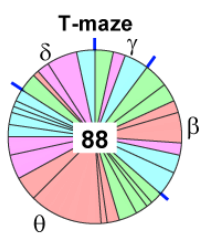

$(P<0.05 / 888)$

$=$ PFC-dHC

$=$ PFC-vHC

$=$ PFC-MD

$=\mathrm{vHC}-\mathrm{dHC}$

- Correct - Incorrect lit

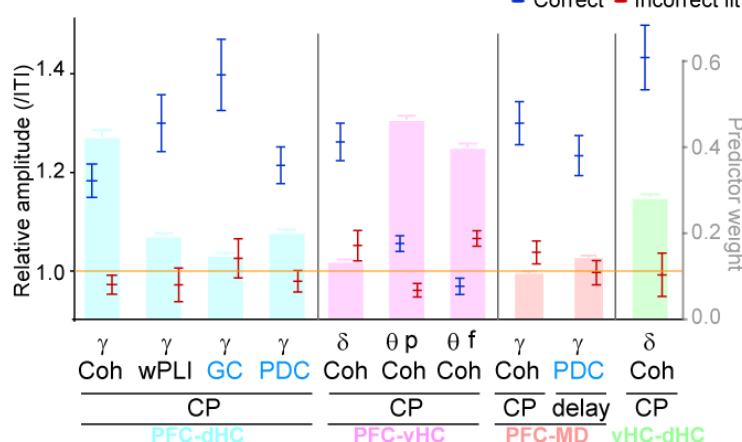

e
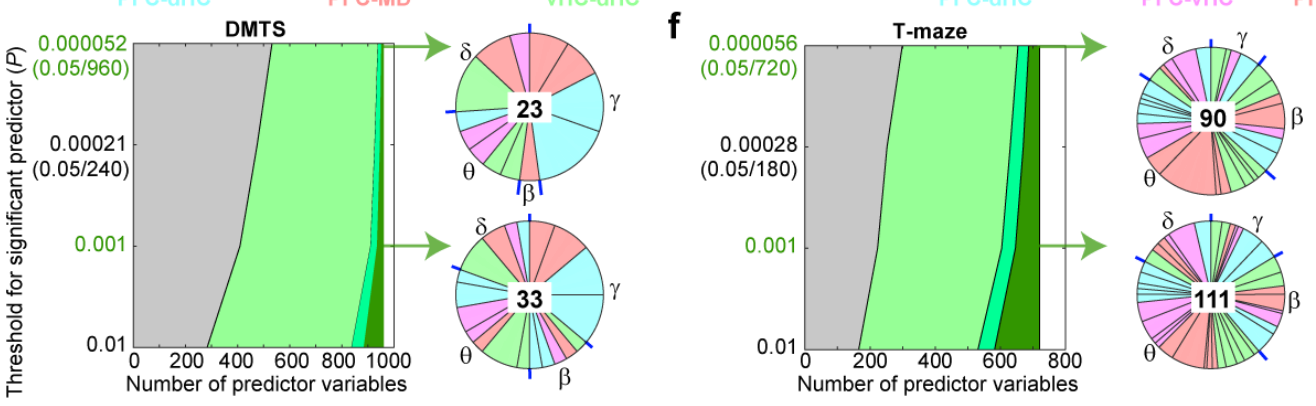

Criterion

- ML \& trial-type

ML (weight)

$=$ trial-type

- never significant

Connection

I PFC-DIMC

$=$ PFC-MD

$=$ PFC-MD

Fig. 4 | Individual connectivity measures predicting WM choice in mice. (a) Matrix

showing all connectivity predictor variables that contributed to the connection-based classifiers shown in Fig. 3b,c. Variables that were significantly associated with WM- 
incorrect CP ( $t$-tests, Bonferroni-adjusted for total number of variables, see (b)) in any of the

three WM tasks are indicated by the corresponding colour, remainder in grey (white squares

have no corresponding variable). Variables from the pre- and post-delay in the 5-CSWM are

combined in single lines. For $\theta$, mean amplitude $(m)$, peak amplitude $(p)$ and frequency of

peak (f) are shown, while for all other frequency bands only the mean amplitude is used due

to the absence of a clear singular peak. At the bottom, all task phases are combined and

only connectivity metrics that are predictive in all three tasks (in at least one phase) are

indicated in black. (b) Share of each connection (coded by colour) and frequency band (stated around pie chart with separations in blue) among all significant predictors ( $N$ stated in centre) for the indicated task. (c-d) Values of absolute (c) or relative (d) predictor variables for DMTS WM (extracted from (a)) in correct (blue) and incorrect (red) trials (left axes). Bars weights normalized within each classifier (i.e., connection) coded by their colour. Significance is not indicated as it applies to all shown variables. Blue font, directional connectivity in the opposite direction compared to connection-name. Error bars, s.e.m. (e, f) Left: Number of variables identified as significant based on ML predictor weight (light green), difference between correct and incorrect trials (medium green), or both (dark green) in dependence on the $P$-value adjustment (y-axes) in the DNMTS (e) and T-maze (f) tasks. Right: Share of predictor variables as depicted in (b) for the $P$-levels indicated in green.

317 Given the prominence and high predictor weights of CP parameters (Fig. 4c-d) - which align with the arising $\gamma$-band connectivity immediately after the CP-poke (Fig. 2a-d) - we wondered 319 if the predictability of WM-choice (Fig. 3b) actually relied mainly on identifying a representation of anticipated reward. Therefore, we replicated the decoding analysis for $S P$ 321 choices for which animals also expect reward. For the SP, however, average decoding 322 accuracies were - although still above the 50\% chance level - considerably smaller, namely 64-68 \% and 54-59\% for predictions based on connectivity and local activity, respectively 
324 (Supplementary Fig. 6a). While this result shows that the attentional element of the task is

325 more difficult to predict from the available parameters than WM choice, it also demonstrates

326 that the obtained $C P$ prediction accuracy was not simply based on representations of motor-

327 action (hole-poking), attention, or reward anticipation. We also repeated the decoding

328 analysis for CP choice with complete omission of all CP parameters. Even though average

329 decoding accuracies decreased significantly for some connections, including the most

330 predictive ones (PFC-dHC, 72\%, PFC-MD, 73.9\%) - but not for vHC-dHC (72\%) - overall

331 accuracies remained far above those obtained from classifiers trained on shuffled labels,

332 and hence above chance level $\left(P<10^{-30}\right.$ and $P<0.002$ for classifiers trained on connectivity

333 or local data, respectively; Supplementary Fig. 6b). Overall, these analyses demonstrate that

334 activities along distinct connections and in distinct frequency bands represent encoding (SP),

335 maintenance (delay), and recall (CP) of WM contents in the 5-CSWM task.

336 WM-related functional connectivity is highly task-specific in mice

337 To investigate if such phase-specific connectivity generalizes across tasks, we performed

338 the same analysis for the classifiers predicting performance in the operant DNMTS and the

339 T-maze assays. In both cases, considerably more parameters carried WM-related

340 information than in the 5-CSWM task (Fig. 4a-b). Compared to DMTS WM, T-maze

rewarded alternation choice was predicted by a much larger number of predictors, with a

342 prominence of $\theta$ - and $\beta$-range (as opposed to $\gamma$-range) variables, and a considerable

343 proportion of SP-parameters (Fig. 4a-b). This pattern is reflective of the diversity of

344 connectivity parameters that have been associated with T-maze performance in prior studies

345 (Supplementary Table 1), and the relatively detrimental effects of optogenetic manipulations

346 in the SP and delay, as compared to the $\mathrm{CP}^{33,34,46}$.

347 Most astonishingly, the combined analysis of all three tasks revealed that none of the

348 specific connectivity parameters identified in one task bore significant predictive power in the

349 other two tasks, revealing a remarkable task-specificity of such parameters (Fig. 4a). It is,

350 however, possible that this finding is simply caused by a very conservative Bonferroni- 
adjustment of the $P$-value used as significance threshold $(0.05 /$ number of all connectivity and activity variables combined; $0.05 / 1184$ for the 5-CSWM, $0.05 / 888$ for the T-maze and 2CSWM). To test this possibility, we repeated the above analysis while relaxing this adjustment incrementally over four orders of magnitude (Fig. 4e-f). However, the number of identified significant parameters, the relative contribution of different frequency bands, and especially the extreme sparseness of overlap between task-specific predictors changed relatively little (Fig. 4e-f, Supplementary Fig. 7-8). This analysis also revealed that far more connectivity parameters are identified according to their prediction weight than according to their amplitude difference between correct and incorrect trials (Fig. 4e-f). This suggests that the classical approach of correlating behavioural performance with the amplitude of a given metric (Supplementary Table 1) likely misses a sizable proportion of WM-related functional connectivity.

To investigate potential differences or similarities between the time-course of individual parameters during the task, we extracted those spectral connectivity parameters that were predictive across all three assays albeit in different phases: directed dHC $\rightarrow$ PFC $\gamma-$ connectivity and intra-hippocampal $\delta$-range coupling (Fig. 4a). Inspection of the time-course of these parameters over the delay and CP revealed that they behaved rather differently in the individual tasks: $\mathrm{dHC} \rightarrow \mathrm{PFC} \gamma$-connectivity showed a transient increase during the delay of all three tasks, but only in the operant tasks a second increase occurred immediately after correct choices (but not after incorrect choices; Fig. 5a-b). Intra-hippocampal $\delta$-coupling even showed a different time course in every task, including a correct choice-specific decrease in the 2-CSWM delay which contrasted sharply with a steady rise during the Tmaze delay (Fig. 5a-b). Thus, even within the few predictor variables that are relevant across all tasks, the actual physiological activity relating to the behaviour differed markedly.

375 Given these results, we directly tested the hypothesis that distinct activity patterns underlie the different rodent WM tasks by rendering task-type a dependent variable: we trained 377 classifiers to decode which one of the three tasks a subject is currently conducting using 
bioRxiv preprint doi: https://doi.org/101101/2021.04.20.440621; this version posted April 21, 2021. The copyright holder for this preprint (which was not certified by peer review) is the author/funder, who has granted bioRxiv a license to display the preprint in perpetuity. It is made available under aCC-BY 4.0 International license.

a

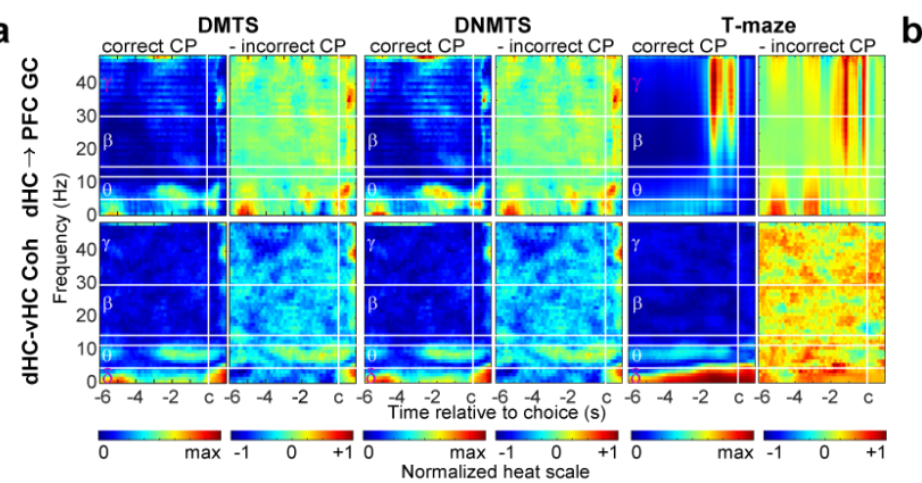

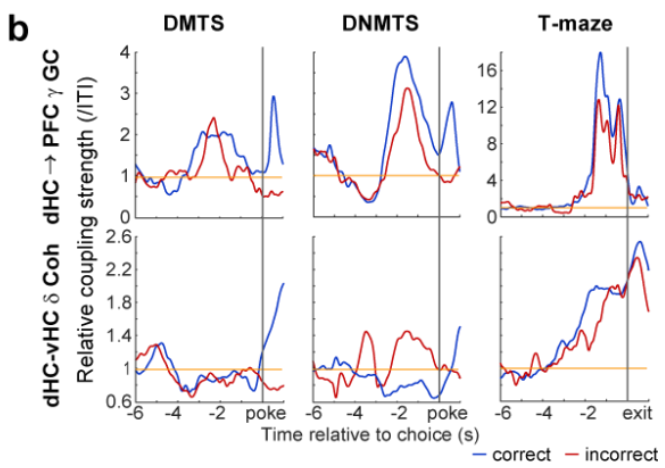

C
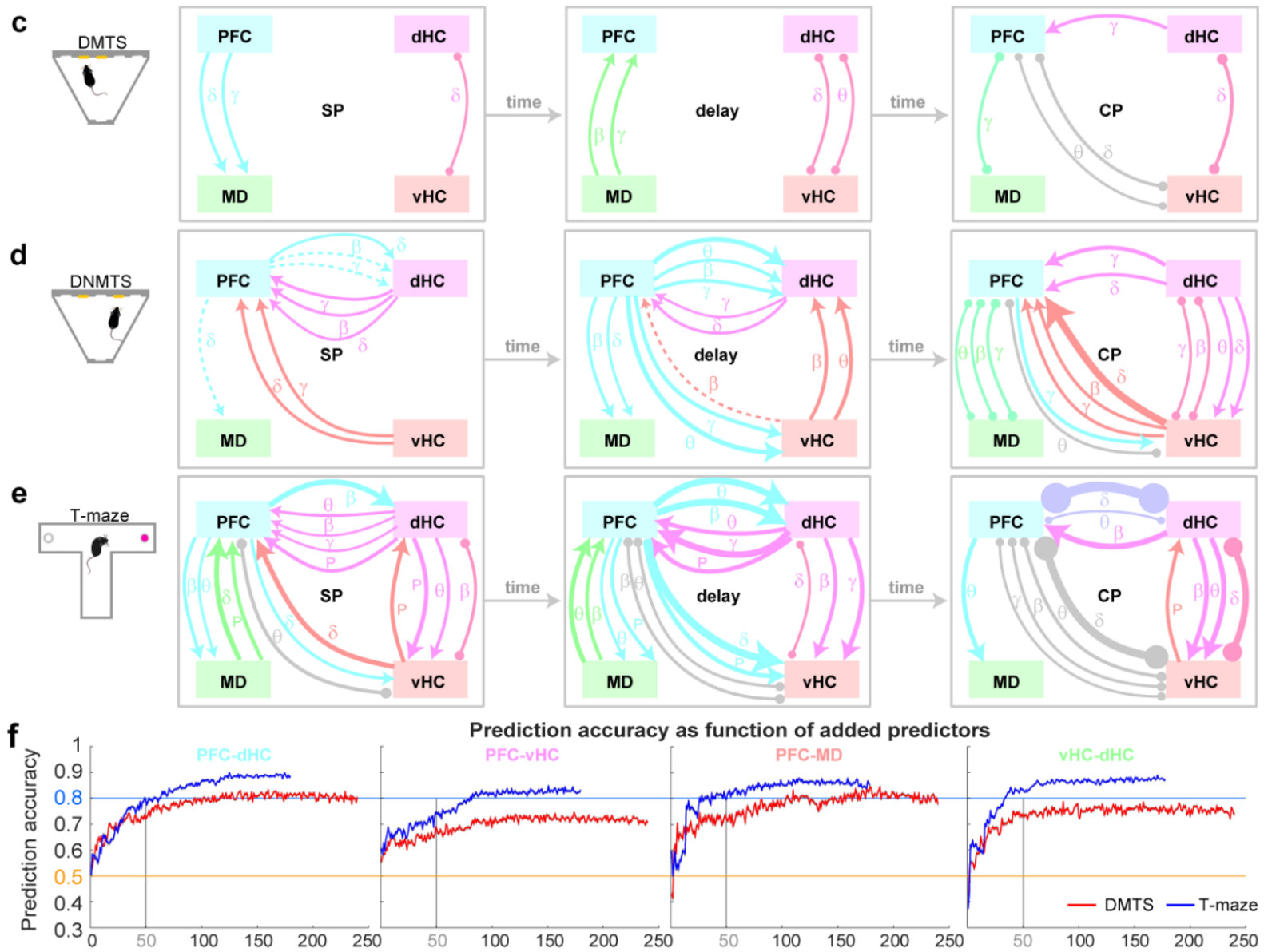
Number of added predictor variables (in order of absolute value of prediction weight)

384 Fig. 5 | Individual connectivity measures predicting WM choice in all tasks in ice. (a) 
different phases; named at the top) are shown as absolute values and as difference between correct and incorrect $\mathrm{CP}$ responses, $-6 \mathrm{~s}$ until $+1 \mathrm{~s}$ around the choice; relevant frequency bands are $\gamma$ (top) and $\delta$ (bottom). (b) Average temporal evolution of those metrics in units of their value during the preceding ITI aligned to the choice point in each task (vertical grey line, poke or exit from decision zone) during correct (blue) and incorrect (red) trials. (c-e) coupling (round-ended arcs, coherence or wPLI) during the three phases of each task identified on the left, derived from significant predictor variables shown in Fig. 4a. For connections where there is a directed connectivity metric of the same frequency, a nondirected metric in the same frequency band is not depicted. Line weights indicate the change in connectivity amplitude in the stated phase relative to the preceding ITI (irrespective if the indicated metric was significant as absolute or relative measure). Measures that are significant but where the amplitude in the indicated phase is identical or smaller than in the classifiers calculated with a reduced number of predictor variables are shown as a function of the number of added predictors, whereby the addition was done in order of normalized prediction weight obtained with all variables (Fig. 3b,c) for DMTS (red) and T-maze (blue) and the named connections. Chance level (50\%) and $80 \%$ accuracy are indicated by coloured lines. Shaded area, s.e.m.

\section{Common WM-related connectivity patterns shared across rodent tasks}

406 To extract commonalities of connectivity between the three tasks, we aggregated predictive 407 non-directed (coherence, wPLI) and directed (GC, PDC) metrics (extracted from Fig. 4a) and 408 depicted their amplitude increases relative to the preceding ITI for each task phase (Fig. 5c409 e). For the T-maze (the only task for which prior reference data exists), this revealed several 410 connectivity patterns associated before with rewarded alternation performance, including 411 vHC-PFC ${ }^{33}$ and dHC-PFC ${ }^{34}$ coupling during encoding and MD $\rightarrow$ PFC $\beta$-range activity during 412 maintenance across the delay ${ }^{14,46}$. Importantly, MD-PFC $\beta$-range delay activity was also 
413 seen in the other two WM tasks, although their directionality differed (MD $\rightarrow$ PFC in the DMTS

414 task; PFC $\rightarrow$ MD in the DNMTS task; Fig. 5c-e). Likewise, further task-independent

415 connectivity patterns emerged in this analysis: prominent vHC-PFC $\delta / \theta-$ coupling and vHC-

$416 \mathrm{dHC} \delta$-coupling in the CP, and MD-PFC $\delta$ coupling in the SP (but with task-dependent

417 directionality; Fig. 5c-e). Some further patterns were shared only by the two DNMTS tasks,

418 e.g., directed PFC-dHC $\beta$ - and $\gamma$-connectivities in the SP and delay (Fig. $5 d$-e). At the same

419 time, this analysis also confirmed that the vast majority of WM-related connectivity was task-

420 specific, especially when comparing the 5-CSWM DMTS to the other two tasks (Fig. 5c-e).

421 An important aspect of this analytical approach is that none of these individually highlighted

422 connectivity measures (Fig. $4 a, 5 c-e)$ is particularly predictive on its own: When performing 423 decoding analysis with reduced sets of predictor variables - starting with the parameter with 424 the single highest weight and adding variables incrementally - the inclusion of several dozen 425 predictor variables was necessary to achieve maximum decoding accuracy (Fig. 5f).

Predictive power of local activity in a single area varies by task phase and type Local oscillatory activity in the four analysed regions also allowed considerable prediction accuracy in all three tasks - sometimes even exceeding that obtained from connectivity metrics (Fig. 3b-c). Therefore, to reveal WM-related local activity metrics, we repeated the prior weight-based analysis for the respective variables (power, local PAC). In the 5-CSWM DMTS task only CP parameters, mostly in the $\beta / \gamma$-range, were significantly associated with WM (Fig. 6a). For the two other tasks, in contrast, significant predictors came from all three phases and were somewhat less frequency-specific; the power of $\mathrm{dHC}$-oscillations across all frequency bands and phases constituted the most prominent cluster of choice-predictors in both assays (Fig. 6a). In agreement with the high decoding accuracy obtained with local activity (as opposed to connectivity) in the DNMTS 2-CSWM (Fig. 3b), many more significant local predictor variables were found for this task compared to the other two, irrespective of $P$-value threshold (Fig. 6b). Importantly, however, there was again hardly any overlap 
bioRxiv preprint doi: https://doi org/10.1101/2021.04.20 440621; this version posted April 21, 2021. The copyright holder for this preprint (which was not certified by peer review) is the author/funder, who has granted bioRxiv a license to display the preprint in perpetuity. It is made available under aCC-BY 4.0 International license.

440 activity parameters had higher amplitudes in correct trials compared to incorrect trials, this

441 was not the case for the T-maze, where virtually all predictive hippocampal activity was

442 lower in correct trials compared to incorrect trials - only PFC and MD power were higher in 443 correct trials (Fig. 6c-d). Hence, as observed in inter-regional connectivity, local activities

444 related to WM-choice were highly task-specific in multiple respects.
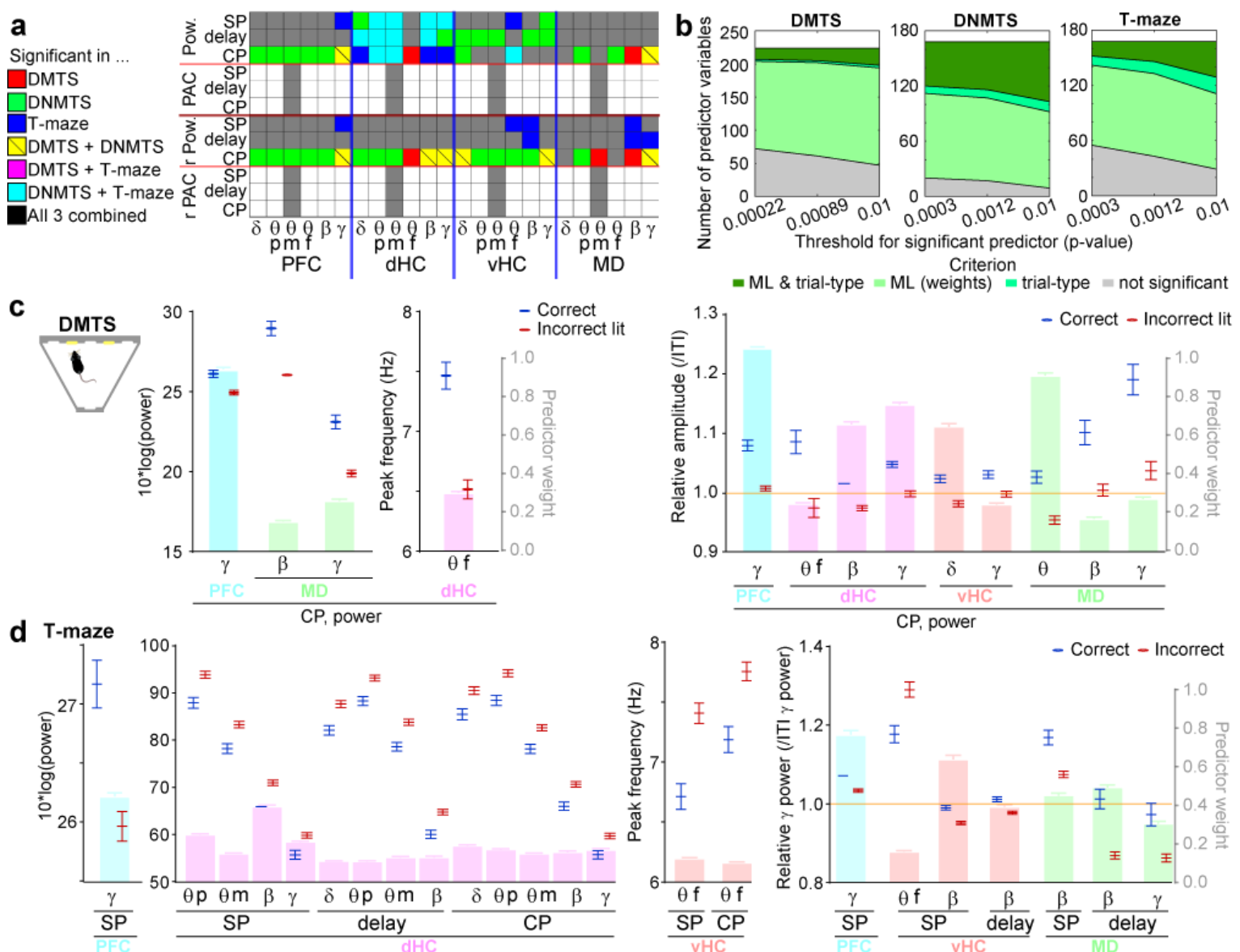

Fig. 6 | Local activity measures predicting WM choice in all tasks. (a) Display and analysis as in Fig. 4a, but for all local activity parameters that contributed to the regionbased classifiers shown in Fig. 3b,c. Variables that were significantly associated with WMperformance according to both their prediction weight and differences between correct and incorrect CP (t-tests, Bonferroni-adjusted for total number of variables) in any of the three WM tasks are indicated by the corresponding colour, remainder in grey (white squares have no corresponding variable). (b) Number of variables identified as significant based on ML predictor weight (light green), difference between correct and incorrect trials (medium 
green), or both (dark green) in dependence on the $P$-value adjustment (x-axes) in the named tasks. (d-e) Values of absolute (left) or relative (right) predictor variables for DMTS (e) or Tmaze (f) WM (extracted from (a)) in correct (blue) and incorrect (red) trials (left axes). Bars in the background, referenced to by right axis, show absolute values of average predictor weights normalized within each classifier (i.e., connection) coded by their colour.

Significance is not indicated as it applies to all shown variables. Error bars, s.e.m. neural activity in humans

462 It remains unclear if highly task-specific and widely distributed WM correlates are only found 463 in rodents or also in human WM. To clarify this question, we used a dataset of intracranial 464 LFP (iEEG) recordings made in 8 human subjects from three sites - PFC, OFC, and MTL during three types of WM assays whose trials were intermixed within a single test session: identity-related WM (differentiating between identical and novel shapes), spatial WM, and temporal WM (remembering the temporal order of two stimuli; Fig. 7a-b) ${ }^{20}$. For each of the three tasks, we applied the same ML-approach as in mice, generating classifiers that use activity data from four phases (SP, pre-cue- and post-cue delay phases, CP; see task schedule in Fig. 7a) from only a single connection or region at a time.

Average decoding accuracies for trial-by-trial prediction of WM-choices were higher than

472 those achieved in mice, ranging consistently between $87-90 \%$ for predictions based on 473 connectivity and between $72-82 \%$ for predictions based on local activity, whereas 474 "predictions" based on shuffled control data remained significantly lower $\left(P<10^{-40}, t\right.$-tests) and were not different from chance level (50\%; Fig. 7c). We also trained classifiers on the combined data from all three inter-regional connections and three regions - either separately

477 for each task-type or combining all types of trials indiscriminately. For task-specific 478 classifiers, average decoding accuracies reached $87.6 \%, 90.8 \%$, and $89.8 \%$ for identity479 related, spatial, and temporal WM, respectively, i.e., no higher than what could be achieved 480 by connectivity data from the single best connection in each task (Fig. 7d). However, 
bioRxiv preprint doi: https://doi org/10.1101/2021.04.20 440621; this version posted April 21, 2021. The copyright holder for this preprint (which was not certified by peer review) is the author/funder, who has granted bioRxiv a license to display the preprint in perpetuity. It is made available under aCC-BY 4.0 International license.

481

482

483

484

485

486

487

488

489

a

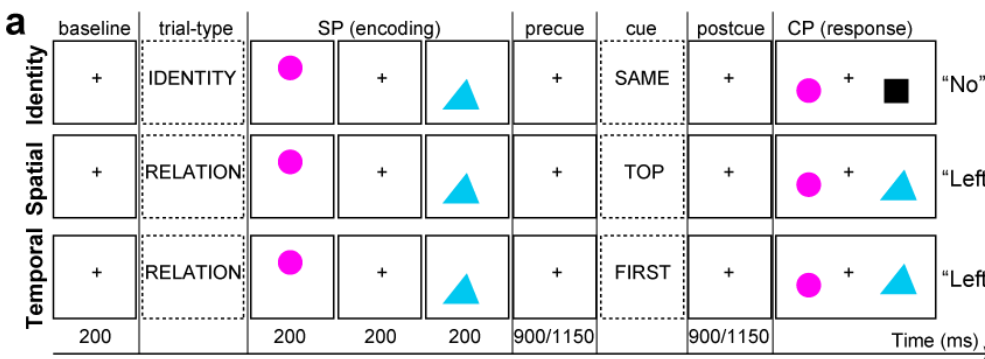

.

WM paradigm

- identity | real

- - spatial $\mid \begin{aligned} & \text { real } \\ & \text { - temporal }\end{aligned}$

........ identity | shuffled

......... temporal

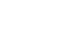

\section{(2) (1) PFC-MTL}

Rank of accuracy in ...

Oidentity WM

spatial WM

Otemporal wM
(4): 4

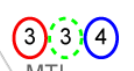

(4):(4) OFC

PFC (3)
490
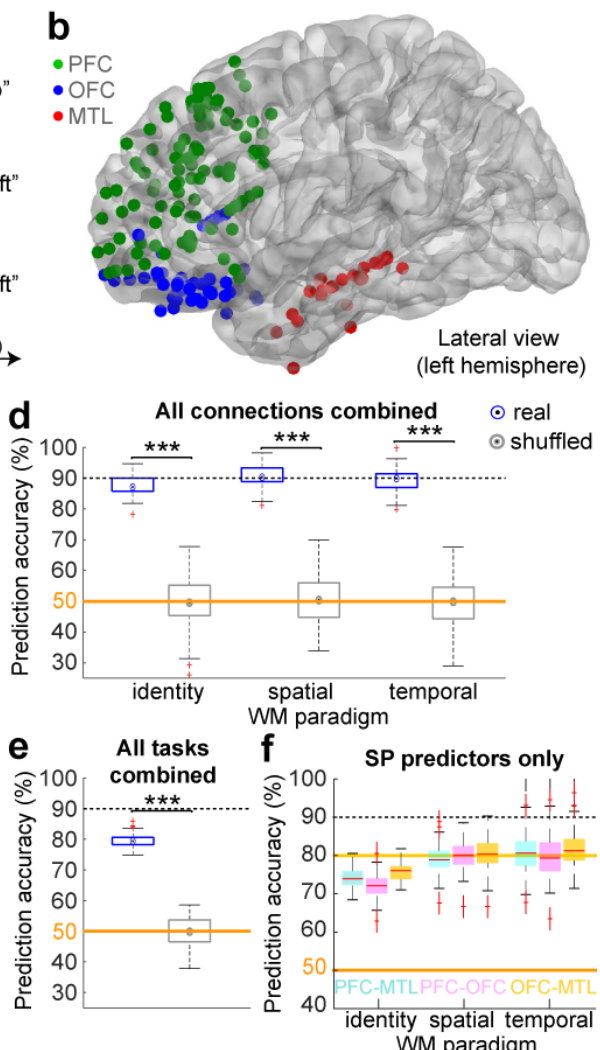

Fig. 7 | Single-trial based prediction of WM choice in humans. (a) Display of the structure of the three human WM tasks, according to ref. ${ }^{20}$ (see Methods). (b) Placements of electrodes as projected onto the left hemisphere, irrespective of actual hemisphere of each electrode. (c) Cross-subject decoding accuracies achieved on average when using connectivity or local activity parameters of the indicated connections (black) or areas (grey), 
bioRxiv preprint doi: https://doi org/10.1101/2021.04.20.440621; this version posted April 21, 2021. The copyright holder for this preprint (which was not certified by peer review) is the author/funder, who has granted bioRxiv a license to display the preprint in perpetuity. It is made available under aCC-BY 4.0 International license.

respectively, to predict WM-based correct vs. incorrect choices in the tasks coded by colour on the left. Accuracies achieved by classifiers trained with randomly shuffled labels are shown as coloured dotted lines; they are consistently lower than accuracies achieved by classifiers trained with real labels in all tasks and connections/regions $\left(P<10^{-40}, t\right.$-tests, not indicated) and assume chance level (50\%, orange). Accuracies of $80 \%$ (yellow line) and 90\% (black dotted line) are indicated to aid comparison. Shaded area represents s.e.m. Numbers in circles colour-coded for the respective paradigm indicate the rank of the average decoding accuracies achieved using data from the respective connection or region. Ranks have been generated from pairwise comparisons with Tukey post-doc tests conducted after significant effects of connection/region in one-way ANOVAs $(P<0.0001$ in all cases); connections/regions that were not significantly different from each other were assigned the same rank. (d) Decoding accuracies achieved when using the classifiers trained on predictors from all connections/regions combined (e) Similar analysis as (d) but with trials from all three paradigms inter-mixed. Blue and grey in (d-e) depicts performance of classifiers trained on trials with correct or incorrect (shuffled) labels (stars indicate $t$-test comparisons between them). Error bars, data range without identified outliers which are highlighted in red; boxes, range between $25^{\text {th }}-75^{\text {th }}$ percentile; dot, median. (f) Similar analysis as in (d) but using only SP variables from the connection indicated by colour as predictors. Red lines indicate mean, boxes the $25^{\text {th }}$ and $75^{\text {th }}$ percentile, whiskers indicate data range without outliers, and red crosses indicate outliers. See also Supplementary Fig. 10 for analysis but using only predictors from single task-phases.

\section{WM-related functional connectivity is highly task-specific and broadly}

\section{distributed in humans}

In order to identify possible correlates of WM in humans, we analysed the prediction weights of the individual connectivity metrics similarly as for the mouse dataset, again extracting WM-related metrics based on the two criteria of prediction weight and a different amplitude of the metric in correct trials compared to incorrect trials (Bonferroni-adjusted $t$-tests). As in mice, WM-related measures (185 out of 1344 connectivity predictor variables) were widely 
524 distributed across connections, frequency bands, and metric types. When inspecting the

525 matrix of significant predictors more closely, some regularities emerged (Fig. 8a): First, WM-

526 related activity was highly task-specific with $88 \%$ of significantly WM-related connectivity

527 metrics being relevant in only a single paradigm. Only a single metric was predictive in all

528 three paradigms - OFC $\rightarrow$ PFC post-cue delay $\gamma$-PDC. The principle task-specificity was

529 maintained also with relaxed $P$-value thresholds (Supplementary Fig. 11-12). Second, by far

530 the most - and the most common - predictors emerged in the $\gamma$-band, irrespective of

531 significance threshold (Fig. 8a-b, Supplementary Fig. 11, 13-14). The $\delta$-band - in contrast to

532 mice - contributed almost no WM-related variables (only one each in spatial and temporal

533 WM, confined to the OFC-MTL connection). Also, the $\theta$-band bore relatively few WM-related

534 connectivity parameters, and these were mostly relevant for spatial WM and to a lesser

535 extent for identity WM, but hardly for temporal WM. Furthermore, $\theta-\gamma$-PAC appeared rather

536 relevant (as found in the same data before ${ }^{20}$ ) in all three types of tasks, especially identity-

537 related WM. Third, changes of a metric relative to the ITI before each trial were rarely

538 predictive. Finally, despite the relatively high decoding accuracy achieved for temporal WM

539 (Fig. 7c-d), the number of connectivity metrics related to this WM-type was considerably

540 smaller (18 out of 1344 measures) than for the other two (72 and 95) and there was hardly

541 any overlap between these metrics and those relevant for the other two WM-paradigms (only

5423 each, mostly in the $\gamma$-band; Fig. 8a-b). In summary, the analysis in humans confirms the

543 high task-specificity, and broad anatomical and frequency-range distribution of WM-related

544 neural activity already seen in mice. 
bioRxiv preprint doi: https://doi.org/10.1101/2021.04.20.440621; this version posted April 21, 2021. The copyright holder for this preprint (which was not certified by peer review) is the author/funder, who has granted bioRxiv a license to display the preprint in perpetuity. It is made available under aCC-BY 4.0 International license.

a

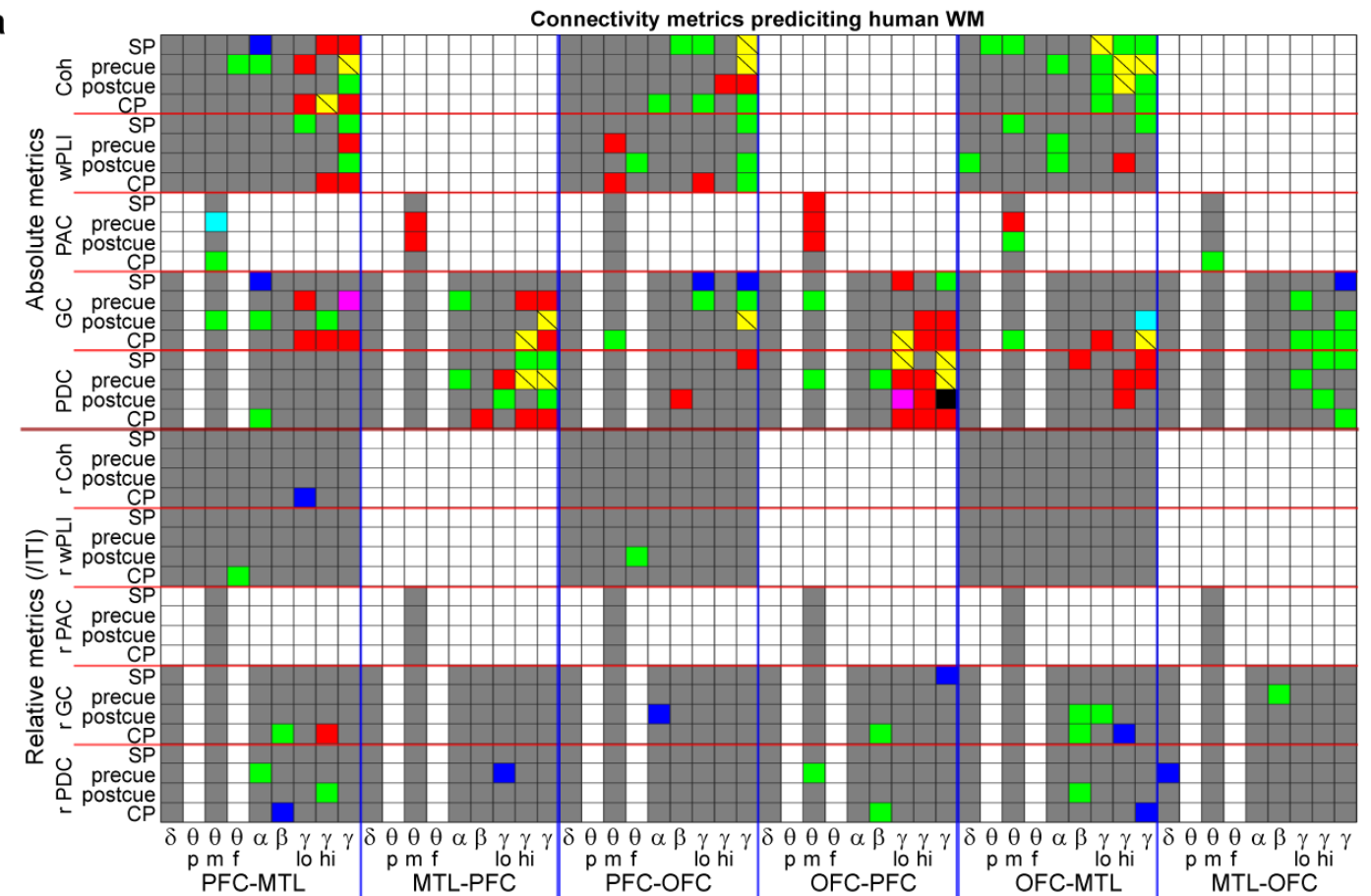

Significant in ...

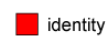

$\square$ spatial

temporal

$\nabla_{+ \text {spatial }}^{\text {identity }}$

$\square$ identity +

$\square$ spatial +

spatial +
identity +

temporal

none

$\square$ no metric
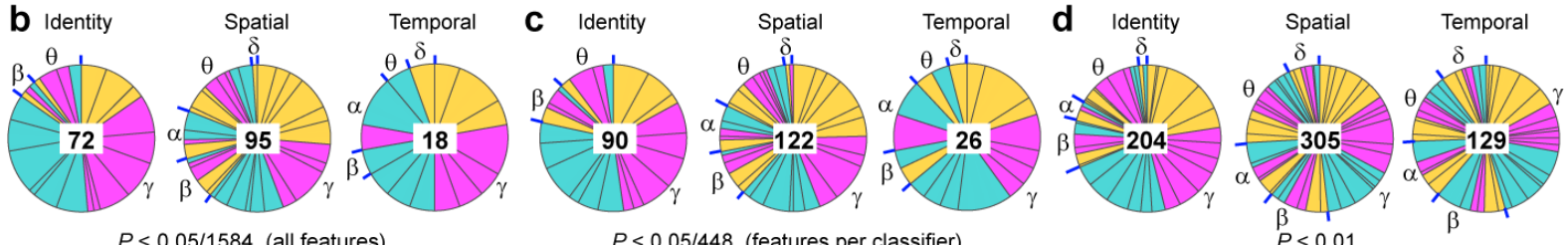

$P<0.05 / 448$ (features per classifier)

e

Identity

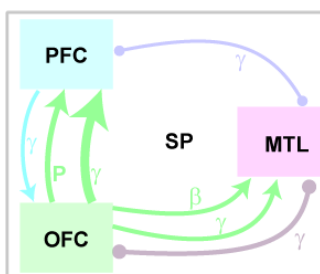

f

Spatial

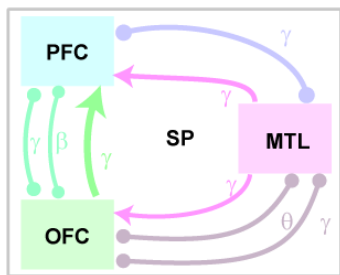

g

Temporal

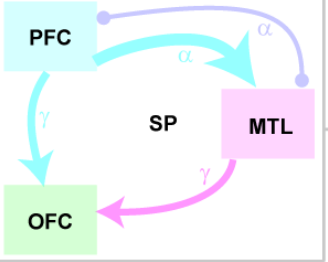

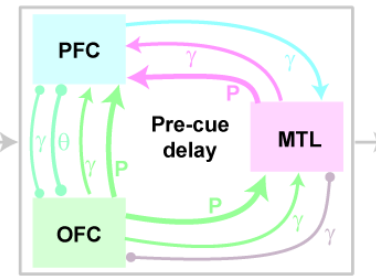

PFC

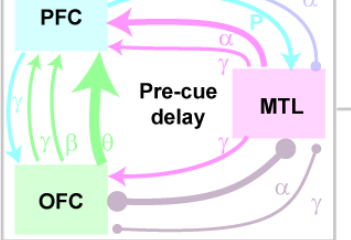

PFC

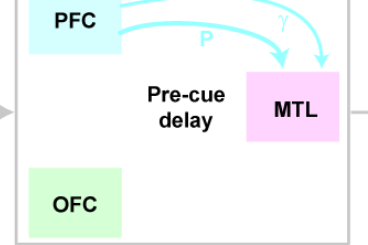

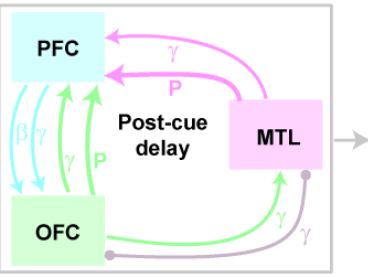
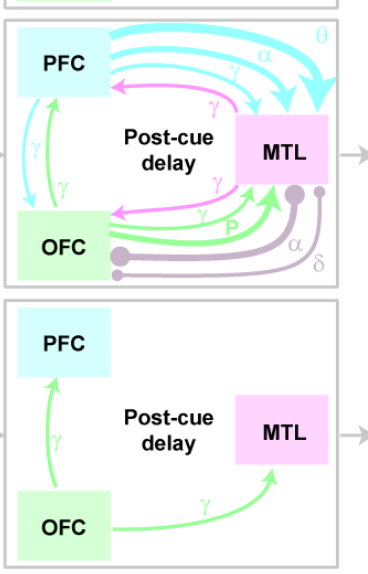

$=\mathrm{PFC}-\mathrm{MTL}=\mathrm{PFC}-\mathrm{OFC} \backsim$ OFC-MTL

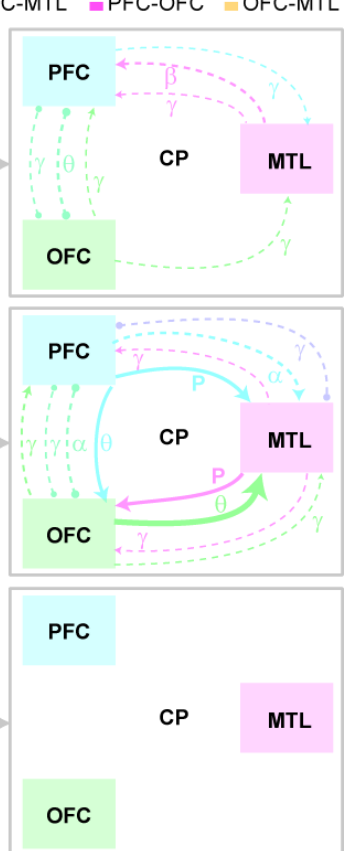

Fig. 8 | Highly task-specific and broadly distributed correlates of human WM. (a) Matrix

547 showing all connectivity predictor variables that contributed to the classifiers shown in (8d) 
bioRxiv preprint doi: https://doi org/10.1101/2021.04.20.440621; this version posted April 21, 2021. The copyright holder for this preprint (which was not certified by peer review) is the author/funder, who has granted bioRxiv a license to display the preprint in perpetuity. It is made available under aCC-BY 4.0 International license.

549

550

551

552

553

554

555

556

557

558

559

560

561

562

563

564

565

566

567

568

569

570

571

572

573

574

575

differences between correct and incorrect CP (see Results) in the paradigms coded by colour (see legend on the right). For $\theta$, mean amplitude $(m)$, peak amplitude ( $p)$, and frequency of peak (f) are shown, while for all other variables only the mean amplitude is used. The $\gamma$-band contributed three predictors each as this frequency was split into a highand low- $\gamma$-range in addition to using the whole range $(30-100 \mathrm{~Hz})$. See Supplementary Fig. 11 for the same analysis with relaxed $P$-value correction and Supplementary Fig. 13-14 for prediction weights of the same variables. (b-d) Share of each connection (coded by colour) and frequency band (stated around pie chart with separations in blue) among all significant predictors ( $N$ stated in centre) for the indicated task. The significance threshold has been Bonferroni-adjusted either by the total number of predictor variables from all connections and regions (1548, corresponding to analysis in panel (a); b), or by the number of connectivity variables for a single connection (448; c), or a standard threshold of 0.01 was chosen (d). (e-

g) Depictions of directed connectivity (arrows, derived from GC or PDC) or non-directed coupling (round-ended arcs, coherence or wPLI) during the four phases of each task identified on the left, derived from significant predictor variables shown in (a). Line weights indicate the increase of connectivity in the stated phase relative to the preceding ITI. Measures that are significant but decrease in the respective phase relative to the ITI are shown as dashed lines. Significant coupling metrics are not depicted if directed measures are represented in both directions. P, $\theta-\gamma$-PAC.

\section{Common $\gamma$-band connectivity across human WM tasks}

To scrutinize this conclusion, we searched for commonalities between tasks by aggregating predictive non-directed (coherence, wPLI) and directed (GC, PDC) metrics and the multiple measures within the $\gamma$ - and $\theta$-bands (extracted from Fig. 8a), and depicted their amplitude change relative to the preceding ITI for each task phase (Fig. 8e-g), as previously done for the mouse dataset (Fig. 5c-e). In this analysis, OFC-PFC $\gamma$-coupling during encoding and directed OFC $\rightarrow$ PFC/MTL $\gamma$-connectivity during the post-cue delay emerged as common patterns present in every task. There were also more commonalities between spatial and 
identity WM, namely $\gamma$-coupling between all three regions that was elevated throughout

577 encoding and delay phases and then decreased below ITI-levels in the CP (Fig. 8e-f).

578 Strikingly in fact, all significant predictors from the $\alpha-\beta-\gamma$-range in these tasks showed

579 elevated amplitudes during encoding and delay, but decreased amplitudes during the CP,

580 compared to their amplitude in the preceding ITI (Fig. 8e-g). Only $\theta$ and $\theta-\gamma$-PAC predictor

581 variables increased in amplitude during the CP in spatial WM (Fig. 8f). Finally, even with this

582 aggregated analysis, not a single connectivity pattern that was shared between any two

583 tasks emerged outside the $\gamma$-band in any task phase (Fig. 8e-g). This analysis suggests that

584 human WM generally relies on anatomically broad, task phase-specific modulation of $\gamma$ -

585 connectivity between several brain regions irrespective of task, while the engagement of 586 oscillatory coupling in other frequency bands is task-specific.

\section{Discussion}

589 Here we demonstrate that WM-related choices can be predicted trial-by-trial in mice and

590 humans using linear decoding of high-dimensional arrays of LFP-based measures of inter-

591 regional connectivity or local activity (the electome). The high decoding accuracies of around

$59290 \%$ (compared to a chance level of $50 \%$ ) achieved in both species are remarkable

593 considering the spatially coarse nature of the extracted neural signal, the short - often subsecond - data traces used to calculate predictors, the intrinsic variability caused by merging data from all analysed individuals with varying electrode placements, and the lack of precise neuronal information as encoded in spike trains of individual neurons ${ }^{10,21,33,41,47}$. Using the

597 trial-by-trial predictive power of physiological activity as the indicator for its association with

$598 \mathrm{WM}^{42}$, this approach enabled a largely unbiased top-down analysis to reveal an 599 unexpectedly rich pattern of frequency-specific connectivity changes during individual 600 phases of distinct WM assays in mice and humans. 
601 The comparative analysis of multiple WM assays - including those that allow control over

602 basic motivational and attentional parameters - provides a unique advantage in that 603 neurophysiological activity patterns which might be truly relevant to WM may be isolated. In 604 this way, we could reveal MD-PFC $\delta$ - and $\beta$-range coupling during memory encoding and 605 maintenance, respectively, as well as vHC-PFC and vHC-dHC $\delta / \theta$-coupling during retrieval 606 as common connectivity patterns across all three rodent tasks - although the vast majority of 607 connectivity proved to be task-specific (Fig. 5c-e). In humans, $\gamma$-band connectivity across all 608 analysed connections was commonly linked to WM-choice across tasks, while WM-related 609 connectivity in other frequency bands was mostly task-specific (Fig. 8).

610 Against a backdrop of widely varying assumptions about which kind of neural connectivity 611 underlies WM (see Introduction), our analysis was initially motivated by the possibility to 612 extract "true" anatomical and frequency-related WM-correlates using the predictor weights 613 generated by the linear classifiers that decode WM-based choices with high accuracy. Our 614 results, however, refute some implicit key assumptions of this endeavour - and, by 615 extension, of many prior investigations of WM-correlates: First, there is no single - or small 616 set of - anatomical regions or connections, types of directional information transmission, or 617 frequency bands that can be regarded as a unique WM correlate. Indeed, previously 618 suggested "correlates", especially in the rodent literature (Supplementary Table 1), could 619 appear as such only because the sum total of connections and measures investigated in 620 each study was small (streetlight effect), as opposed to the 1184 and 1584 metrics analysed 621 here in mice and humans, respectively. In our study, virtually every analysed frequency 622 band, metric, connection and region bore some predictive power regarding WM-mediated 623 choice.

624 Second, there is no single behavioural WM-task that could be regarded as representative of 625 the generic psychological construct termed "working memory" in order to allow the 626 identification of the neurophysiological correlate of that construct. The latter is illustrated by 627 the enormous variability in the patterns of predictive connections and metrics across task- 
paradigms in both species. In other words, a physiological variable that correlates with

629 choice accuracy in the T-maze represents a neurophysiological correlate of T-maze

630 performance, but not necessarily of WM. The same principle applies to our cross-species

631 comparison, as the uncovered candidates for a generic (task-independent) WM correlate

632 originated from different frequency bands in humans $(\gamma)$ than in rodents $(\delta-\theta-\beta)$. A 633 translational implication of these findings is that it is likely impossible to define 634 neurophysiological underpinnings of "working memory" as a uniform psychological construct.

635 However, the existence of certain tasks that represent such a psychological function (i.e., 636 that engages a physiological mechanism that is central to all WM tasks across species) is an 637 implicit key assumption of the Research Domain Criteria (RDoC) approach, which envisions 638 to use those representative paradigms in search of WM-enhancing cellular and molecular 639 targets $^{48}$. Our data suggest that the key target variable in the preclinical discovery of WM640 enhancing compounds might be the appropriate regulation of $\gamma$-range connectivity (given its 641 importance for human WM) rather than behavioural performance in any particular rodent 642 task.

643 The analysis of the human dataset, in particular, paints a rather different picture of what a 644 correlate of WM could be - at least when searched for in LFP-data. In all three tasks, 645 prediction accuracy calculated from connectivity (as opposed to local activity) metrics was 646 not only very high, but it was also roughly equal between the three analysed connections, 647 even though these are anatomically quite distinct. This was the case even with the limited 648 analysis incorporating only SP or post-cue delay connectivity as predictors of spatial WM. 649 These findings may be taken as an indication that WM-related information is extremely 650 broadly distributed, and - rather than specific activity located in a certain connection or 651 region - it is the ability to manipulate information flow across brain regions as such, that determines task performance ${ }^{49,50}$. This model is in line with the ever growing list of brain areas that are implicated in WM, including the superior frontal ${ }^{51}$, anterior cingulate ${ }^{52,53}$ and sensory cortex ${ }^{54,55}$, ventral tegmental area $^{28}$, and the nuclei of the midline and anterior 
655 thalamus ${ }^{4,9,34}$, and the concept that different areas may be involved depending on the

656 strategy used to solve the task ${ }^{54}$. An unexpectedly broad anatomical representation of

657 sensory and behavioural information across the brain has recently been uncovered by

658 decoding activity of individual neurons in multiple cortical areas ${ }^{56,57}$, and our decoding

659 analysis of LFP-based connectivity in cognition underscores this phenomenon.

660 In conclusion, our multi-area decoding approach and cross-task cross-species comparative

661 analysis revealed not only a rich functional connectivity supporting WM, exceeding the WM-

662 associated connectivity described before. It also demonstrated an unexpected task- and

663 species-specificity of WM-related neural coupling that raises substantial caution regarding

664 the predictive translational value of each assay and demands to re-think our search for

665 physiological WM-correlates. 


\section{Animals and surgery}

All experiments were performed in accordance to the German Animal Rights Law

670 (Tierschutzgesetz) 2013 and were approved by the Federal Ethical Review Committee

671 (Regierungsprädsidium Tübingen) of Baden-Württemberg, Germany (licence number

672 TV1399). The rodent cohort, surgery and histology details have been described in our prior

673 publication of the open-field data from the same cohort ${ }^{37}$. Briefly, 12 C57BL/6N wildtype

674 mice, including 8 males, were selected from offsprings of an in-house colony (heterozygous

675 Gria1 ${ }^{\text {tm1Rsp }}$ mice; MGl:2178057) ${ }^{58}$, group-housed in Type II-Long individually ventilated

676 cages (Greenline, Tecniplast, G), enriched with sawdust, sizzle-nest ${ }^{\mathrm{TM}}$, and cardboard $^{2}$

677 houses (Datesand, UK), and subjected to a $13 \mathrm{~h}$ light $/ 11 \mathrm{~h}$ dark cycle. The mice were

678 implanted with electrodes at ca. 9 months of age, after 2 months of training in the DMTS-WM

679 task and prior habituation training (see below). Single polyimide-insulated tungsten wires of

$68050 \mu \mathrm{m}$ diameter (WireTronic Inc., CA, US) were implanted, with reference to Bregma (in

$681 \mathrm{~mm}$ ), into the PFC (AP +1.8-1.9, ML 0.3-0.35; 1.8-1.9 below pia), MD (AP -1.2, ML 0.3, 2.7

682 below pia), dHC (AP -1.9-2.0, ML 1.5, 1.4 below pia), and vHC (AP -3.1-3.2, ML 2.9-3.0,

$6833.4 \mathrm{~mm}$ for single and 3.8-3.9 $\mathrm{mm}$ for dual electrodes below pia). In most mice, dual

684 electrodes were used for PFC and $\mathrm{vHC}$, whereby the second electrode was placed about

$6850.5 \mathrm{~mm}$ higher than the stated distance from pia. Both hemispheres were implanted at

686 roughly equal proportion. Stainless steel screws $(1.2 \mathrm{~mm}$ diameter, Precision Technologies,

687 UK) were implanted in the contralateral hemisphere ca. $1 \mathrm{~mm}$ from the midline above the 688 cerebellum (AP -5.5) for ground and above the anterior frontal cortex (AP +4.0) for additional 689 reference (used for the analysis in the open-field test, but not for the present analysis), and 690 where connected with a $120 \mu \mathrm{m}$ PTFE-insulated stainless steel wire (Advent Research 691 Materials Ltd., UK; Fig. 1b). All electrode wires were connected to pins in a dual-row 6-pin or 692 8-pin connector (Mill-Max, UK). Electrode placements were determined post-mortem from 693 electrolytic lesions made under terminal ketamine/medetomidine anaesthesia followed by 
694 perfusion-fixation. Misplaced electrodes were excluded, leaving the following number of

695 used electrodes per region; PFC: 12; dHC: 7; vHC: 9; MD: 4. The resulting number of

696 contributing connections were PFC-dHC: 7; PFC-vHC: 9; vHC-dHC: 6; MD-PFC: 4.

\section{Operant DMTS 5-CSWM task}

698 The principal schedule of the task was as previously described ${ }^{45}$ and emulates the combined 699 attention and memory (CAM) task previously developed in rats ${ }^{59}$. In the present study, the 700 task was conducted in custom-designed pyControl-based operant boxes that were optimized 701 for both simultaneous electrophysiological recordings and the acquisition of the 5-CSWM 702 task (operant box design-files and task-scripts implementing all task paradigms used in this study available from https://github.com/KaetzelLab) ${ }^{44}$. The latter was done by using a 5choice poke-wall with a larger distance between the holes $(3 \mathrm{~cm}$ edge-to-edge; $4.5 \mathrm{~cm}$ centre-to-centre), which we chose because of the long learning time in our standard commercial boxes used before ${ }^{45}$. Briefly, each trial of the task is divided into a sample phase (SP), a delay-phase (delay) - which is further sub-divided into a pre-delay and a post-delay by the time point of reward collection - the choice phase (CP), and the inter-trial interval (ITI). In the default state, the task is conducted in the dark (house-light off) with the only illumination deriving from the poke- and receptacle holes in certain task phases. The SP is identical to that of the 5-choice-serial-reaction-time task (5-CSRTT), except that premature responses are not punished: one of the 5 holes in the 5 -choice wall is illuminated for a certain stimulus duration (SP-SD) and mice need to poke into that hole within the limited

714 hole time (SP-SD plus $1 \mathrm{~s}$ ) in order to obtain a small reward ( $20 \mu \mathrm{l}$ or, in stages 2 and

715 onwards, $10 \mu$ strawberry milk) at the receptacle at the opposite end of the wall during the

716 pre-delay time. The reward-collection (receptacle exit) starts the post-delay (2 s in all cases)

717 after which the originally presented hole and one randomly assigned other hole is illuminated

718 (thereby starting the CP) for a certain stimulus duration (CP-SD, $5 \mathrm{~s}$ for all protocols shown

719 in Fig. 1). Mice have to poke into the same hole as in the SP, realizing a DMTS-rule, in order 
or omissions in the SP or CP lead to a $5 \mathrm{~s}$ time-out period (house-light illuminated; reward omitted) and the start of a new trial after an ITI of $5 \mathrm{~s}$. Before training, mice were habituated to the operant box, to consuming the strawberry milk (Müller®, Germany) reward from the receptacle, and to poking into the 5-choice wall to obtain a reward (acquisition of the basic operant cycle).

Subsequently, training was conducted through multiple stages across which the task became incrementally more difficult due to a shortening of SDs and an increase in the number of CP-stimulus configurations (see Supplementary Table 5). During pre-surgery training - in order to compare performance between groups - no performance-based staging was applied, but instead all mice were trained on the stage 1 for the same number of 21 days, and then transitioned through the remaining three training stages (2-4) with $2-3 d$ of training per stage. Parameters defining the stages are found in Supplementary Table 5. continued $\sim 4-5$ weeks after surgery on the baseline stage (4) for 5 weeks to allow the mice to approach asymptotic performance. Subsequently, mice were trained further for 3 weeks in a tethered mode, with the headstage (see below) mounted on their heads, in a baseline protocol with shorter SD in SP (10 s) and CP (5s; stage 5) to increase the number of obtained trials and better standardize the encoding time. Subsequently, mice were taken through three series of challenge protocols with intermittent training on the baseline stage 5 .

740 The same challenge was conducted on 2-3 consecutive days in order to obtain sufficient

741 trials for later analysis. The three series were (a) pure delay challenges, where the pre-delay was extended from 0 to 5 and $10 \mathrm{~s}$, (b) a distraction challenge with an illumination of the house-light for $0.5 \mathrm{~s}$ starting randomly timed between $0.8-1.3 \mathrm{~s}$ of the $2 \mathrm{~s}$ post-delay phase,

744 and (c) a combined attention (SP) and working memory challenge with an SP-SD of $1 \mathrm{~s}$ 745 (instead of the $2 \mathrm{~s}$ of the specific baseline stage 6 of this challenge) and a pre-delay of $5 \mathrm{~s}$, 746 which was preceded by a sole attention challenge (1 S SP-SD, 0 s pre-delay). Most of the 747 analysis shown uses the data from the final (combined) challenge, although decoding 
748 analysis was also conducted for the other challenges in order to replicate the analysis and,

749 additionally, to assess the capacity of cross-prediction between classifiers from entirely

750 different challenge-conditions (Supplementary Fig. 5). The post-delay remained 2 s, starting

751 with the exit from the reward receptacle and spent in darkness, throughout all challenge and

752 baseline protocols.

\section{Operant DNMTS 2-CSWM task}

754 The operant DNMTS task followed the same principal trial-schedule as the 5-CSWM task

755 except for two modifications: the implementation of a non-match-to-position rule (i.e. animals

756 are rewarded for choosing the illuminated CP poke-hole that is not the one, that they poked

757 in the SP) and a simplified set of choice options using always only holes 2 and 4 of the 5-

758 choice wall (making it similar to a task developed by Goto \& Ito ${ }^{60}$ ). Mice were trained in this

759 task only after the T-maze (see below) in order to ease the switching from the prior, opposite

760 task-rule. All mice were trained for 30 training sessions on the 2-choice DNMTS task, then

761 were tested in two delay challenges, in which the pre-delay was extended to 5 and then $10 \mathrm{~s}$

762 (2 $d$ each). A subset was then trained in a 5-choice version of the same task (i.e. the

763 DNMTS-version of the 5-CSWM task) for $12 \mathrm{~d}$ without and for $3 \mathrm{~d}$ with headstage mounted,

764 but performance in many mice was not sufficient, and hence mice were returned to the 2-

765 choice version and trained for a further $5 \mathrm{~d}$ with mounted headstage. The other subset

766 moved directly to $5 \mathrm{~d}$ of training with mounted headstage. Then, recordings were conducted

767 on two days with baseline training and two days each for two delay challenges, in which the

768 pre-delay was extended to 5 and then $10 \mathrm{~s}$. Given the relatively low performance in the delay

769 challenges, the data from the two baseline days was used for further analysis of

770 electrophysiology data. In all protocols, the SP-SD was $8 \mathrm{~s}$, the CP-SD was $5 \mathrm{~s}$, the limited

771 hold time exceeded the SD by $1 \mathrm{~s}$, ITI and time-out were each $5 \mathrm{~s}$, the post-reward delay

772 was $2 \mathrm{~s}$ (spent in darkness), the SP reward was $10 \mu \mathrm{l}$, and the CP reward $60 \mu \mathrm{l}$. 


\section{Non-matching-to-position T-maze rewarded alternation SWM task}

774 Rewarded alternation-based SWM was tested in a T-Maze with transparent walls (10 cm

775 high), intransparent floor (red PVC), and food wells (made from white Teflon®) placed in the

776 end of all three arms. Sliding doors could be used to block off either of the two choice arms

777 in the SP and the initial $10 \mathrm{~cm}$ partition (containing the food-well) of the start arm during the

778 delay and ITI. Before testing mice were habituated to the surroundings and to the condensed

779 milk reward (10\% Ja!-Kondensmilch®, Germany, diluted 1:1 with drinking water) at first

780 cage-wise and later independently. The task schedule was identical to what we previously

781

782

783

784

785

786

787

788

789

790

791

792

793

794

795

796

797

798

799

800 described $^{26}$, involving ten trials per day each consisting of an SP and a CP. During the SP, mice were placed at the beginning of the start arm facing the experimenter, and were left to run into the pseudo-randomly assigned goal arm that was not blocked to obtain a reward. Subsequently, mice returned to the end of the start arm to obtain another, small reward while being enclosed for a $5 \mathrm{~s}$ delay period, during which the door from the previously blocked goal arm was removed. Once the delay had passed, the CP started in which the mouse was allowed to choose between one of the goal arms of which the previously unvisited one was rewarded (correct choice), while the other one was not (incorrect choice). Once the CP was completed, mice were motivated to return to the start arm to obtain another small reward there while being enclosed for an ITI of $20 \mathrm{~s}$ before the next trial commenced. 10 consecutive trials were conducted per day; testing was conducted over $8 d$ with a delay of $5 \mathrm{~s}$ and for another $4 \mathrm{~d}$ with a delay of $30 \mathrm{~s}$ - all 12 sessions were conducted with simultaneous recording of LFP-activity, i.e. a headstage was mounted and tethered through an SPI-cable. In each session, a new sequence of open arms in the SP was used whereby half of the 10 trials were always assigned to the left arm, and the sample arm was not the same for more than three consecutive trials.

\section{Acquisition and pre-processing of mouse data}

Prior to testing, a 32-channel RHD2132 headstage (Intan Technologies, CA, US) was plugged into the implanted connector via a custom-built adaptor that interfaced a 36-pin Omnetics connector (A79022-001, MSA components, G) with another 6-pin or 8-pin Mill-Max 
connector. The headstage was connected to an Open-Ephys acquisition board (https://open-

802 ephys.org, US; obtained through the Open-EPhys store at Champalimaud, Portugal) via two

803 light-weight flexible SPI-cables (Intan Technologies), sometimes daisy-chained through a

804 custom-connected miniature slip-ring (Adafruit, NY, US). The adaptor was wired so that all

805 signals were referenced to the ground-signal obtained from above the contralateral 806 cerebellum. Data were amplified and digitized, sampled at $20 \mathrm{kHz}$ and band-pass filtered at

$807 \quad 0.1-250 \mathrm{~Hz}$ for all subsequent analysis of LFP signals. In operant tasks, all individual task-

808 and behavioural events were recorded by pyControl. Additionally, all events relevant to time-

809 locked electrophysiological analysis of WM task-phases and choices (e.g., correct SP and

$810 \mathrm{CP}$ responses) were encoded as patterns of transistor-transistor logic (TTL) signals by

811 pyControl and recorded as time-stamps with the electrophysiological data by the Open-

812 EPhys acquisition software using the 8 analogue inputs of the acquisition board and a

813 dedicated BNC-HDMI interface board (Open-EPhys). For the T-maze task, ANY-maze

814 (Stoelting) was used to track the position of the animal in the different subdivisions of the

815 maze, and this positional information was encoded in patterns of TTL-signals recorded via

816 an AMi-interface board and a BNC-HDMI interface board as time-stamps with the

817 electrophysiological signals in the Open-EPhys acquisition software.

818

819 Human intracranial electrophysiology data during WM

820 A publicly available dataset of multi-site intracranial recordings during three WM tasks ${ }^{20}$ was 821 downloaded from http://dx.doi.org/10.6080/KOVXODQD. It includes data from 10 adult 822 human subjects (mean \pm SD [range]: $37 \pm 13$ [22-69] y; 7 males) who were implanted with 823 intracranial electrodes to identify epileptic foci for surgical resection. Electrode placements 824 were in the medial temporal lobe (MTL, i.e., CA1; CA3/dentate gyrus; subiculum; or 825 parahippocampal, perirhinal, or entorhinal area), lateral PFC (inferior, middle, or superior 826 frontal area), and OFC (orbitofrontal, frontal polar, or medial prefrontal area). Only subjects 827 with electrodes localized in all three regions were included in the present analyses $(N=8)$. 
828 Details of behavioural testing, data acquisition, and pre-processing have been described

829 previously ${ }^{20}$. In brief, three different WM tasks were conducted, whereby subjects had to

830 either identify a previously indicated object identity, location, or temporal order of two visual

831 stimuli. Trials from all these three tasks were pseudo-randomly mixed from trial to trial in a

832 single test session. Each trial started with a $1 \mathrm{~s}$ pre-trial fixation interval, after which a screen

833 indicated whether in the respective trial would be tested for object identity or spatiotemporal

834 position $(800 \mathrm{~ms})$. Subsequently, the SP started in which two shapes were presented

835 subsequently for $200 \mathrm{~ms}$ each, separated by $200 \mathrm{~ms}$. After a subsequent pre-cue delay (900

836 or $1150 \mathrm{~ms}$, varied pseudo-randomly), a cue appeared for $800 \mathrm{~ms}$ that specified which of the

837 two shapes would need to be identified in the later CP according to a rule of identity

838 (same/different), spatial location (top/bottom) or temporal order (first/second). After a post-

839 cue delay (900 or $1150 \mathrm{~ms}$, varied pseudo-randomly), the CP started as two shapes were

840 presented, of which the participant had to choose the one that was correct according to the

841 prior cue. All trials from all patients were merged for subsequent analyses, just as was done

842 for the mouse WM data. The fully pre-processed data (as described ${ }^{20}$ ) was used for the

843 current analysis.

\section{Data analysis}

845 All signal analyses were done in MatLab (MathWorks). Mouse electrophysiology data were

846 exported to MatLab and, for all LFP analyses, down-sampled to $1 \mathrm{kHz}$ and analysed with

847 custom-written scripts. To reduce low frequency drift, signals were first detrended using the

848 locdetrend function of the Chronux signal processing toolbox (http://chronux.org/) with $1 \mathrm{~s}$ of

849 data and a sliding window of $0.5 \mathrm{~s}$. Trials were excluded from further analyses if the

850 amplitude exceeded the $5^{\text {th }}$ standard deviation within each channel for more than $10 \%$ of the

851 trial duration. In mice, PFC and $\mathrm{vHC}$ were recorded with dual electrodes. In case both

852 electrodes were located in the intended target area (PrL/Cg1 for PFC and fissure for vHC),

853 as inferred from lesion sites, all metrics were calculated for both electrodes and the result

854 was averaged to obtain the final value. For the human dataset, the number of electrodes per 
855 site varied between 1 and 28 per area. Therefore, for connectivity measures, each single

856 metric was calculated for every possible inter-regional pair of electrodes and the resulting

857 value was averaged across all combinations of a single connections. Analogously, for local

858 activity measures, each single metric was calculated for every electrode and the resulting

859 value was averaged across all electrodes of a given area for each subject.

860

\section{Power and non-directional synchrony}

862 Power and coherence spectra were calculated with Chronux routines implemented in the

863 Chronux toolbox using the multi-taper method ${ }^{61}$. Power values were expressed as $10^{*} \log _{10}$

864 values for all analyses and the range of frequencies was set from 0.1 to $48 \mathrm{~Hz}$. A time-

865 bandwidth product of 9 and 17 tapers were used to calculate power and coherence during

866 defined time-periods during ITI, sample phase, delay (if applicable) and choice phase. To

867 address the issue of volume conduction, we calculated the weighted phase lag index

$868(\text { wPLI })^{62}$ using routines implemented in the FieldTrip toolbox ${ }^{63}$. The original trials were further

869 divided into 99\% overlapping "pseudo trials" with a length of 600 ms and padded to the next

870 power of two. The complex cross-spectrum was computed using a Hann taper with a

871 spectral smoothing of $2 \mathrm{~Hz}$. Time-frequency spectral analyses were performed with routines

872 from the FieldTrip toolbox using Morlet wavelets with a width of 3 cycles steps of $10 \mathrm{~ms}^{63}$.

873 Time periods before and after the time frame of interest were padded with real data to avoid

874 artifacts of too long wavelets at low frequencies.

\section{Directional Synchrony}

876 Non-parametric Granger Causality (npGC) $)^{64}$ and partial directed coherence (PDC) $)^{65}$ were

877 calculated using the FieldTrip toolbox ${ }^{63}$. The original trials were further divided into $50 \%$

878 overlapping "pseudo trials" with a length of 1 s and padded to the next power of two, differing

879 from power and non-directional synchrony measures because 99\% overlap did not provide

880 substantially different results but came with a much higher computational effort. The complex

881 cross-spectrum was computed using a Hann taper with a spectral smoothing of $2 \mathrm{~Hz}$. The 
882 noise covariance matrix and transfer function were obtained by applying Wilson's spectral

883 matrix factorization to complex Fourier-spectra. This non-parametric approach was shown to

884 be better at capturing all spectral features, less error prone because no model order had to

885 be chosen, computationally faster than applying autoregressive modelling ${ }^{66,67}$, and to deliver

886 virtually same results when used on our LFP data ${ }^{37}$. Time-frequency representations of $887 \mathrm{npGC}$ and PDC were obtained by Morlet wavelets using the same configurations as 888 described above.

890 Cross-frequency coupling $(\mathrm{CFC})^{68}$ was assessed using the measure of phase-amplitude coupling (PAC), the statistical relationship between the phase of a low-frequency and the amplitude of a high-frequency component, in a cross-regional analysis ${ }^{69,70}$. Time series data were first band-pass filtered in the desired frequency ranges, followed by a Hilbert transform

894 using the MatLab function hilbert to calculate the real and imaginary parts of the signal to obtain the instantaneous amplitude and phase. For each trial, intra- and inter-regional PAC were determined by calculating the modulation of $\gamma$-amplitude by $\theta$-phase using the phaselocking technique proposed by Voytek et al. with routines described in ${ }^{71}$.

\section{Supervised Machine Learning}

899 To validate the calculated measures of neural connectivity and to identify predictive variables

900 we employed supervised machine learning algorithms. Spectrally resolved parameters (e.g.,

$901 \gamma$-coherence, $\gamma$-power) from each inter-regional connection (e.g., dHC-PFC) and local brain 902 region (e.g., dHC) were analysed separately using different classifiers. For classification, we 903 used the absolute parameter values as well as the ratio of each parameter relative to the 904 preceding ITI. In mice, 240 connectivity metrics per connection and 56 local activity metrics 905 per region were used in the 5-CSWM DMTS task (1184 for all connections and regions 906 combined; 296 per task-phase), and 180 connectivity metrics and 42 local activity metrics 907 were used on the operant 2-CSWM DNMTS task and the T-maze (888 combined). The 908 difference originates from the usage of 4 task phases, including pre- and post-delay phases, 
in the 5-CSWM task, but only 3 phases (one delay phase) in the two DNMTS tasks, which

910 was the post-delay in the 2-CSWM task. For all predictor matrices, the pre- and post-delay

911 phases were combined to allow uniform comparisons between all three tasks. In humans,

912448 connectivity metrics per connection and 80 local activity metrics were generated for the

913 analysis shown in Fig. 7, using all 4 task phases including SP, pre-cue delay, post-cue

914 delay, and CP (classifiers omitting the CP were also calculated, see Supplementary Fig. 10).

915 Note that, in humans more predictors arise because frequency bands have been determined

916 additionally in the alpha-band $(8-12 \mathrm{~Hz})$, and in a higher $\gamma$-band $(50-100 \mathrm{~Hz})$ in addition to

917 the low $\gamma$-band $(30-49 \mathrm{~Hz})$ used in rodent analysis. We also included the combined band (30-

$918100 \mathrm{~Hz}$ ) as separate predictor. To ensure a sufficient number of trials for classification and

919 the general validity of the identified predicting variables, we merged data from all subjects of

920 one group, i.e., from all mice provided correct electrode placement (see Supplementary

921 Table 6) or from the subset of human subjects with coverage of all three regions,

922 respectively.

923 Since rodent and human subjects performed proficiently above chance level resulting in 924 more correct than incorrect trials ${ }^{20}$, we used a synthetic minority over-sampling technique 925 (SMOTE) to construct a balanced dataset with five nearest neighbours to consider ${ }^{72}$. All electrophysiological predictor variables of a classifier were normalized between 0 and 1 , 927 setting the maximum empirical value for each metric to 1 . We used $90 \%$ of the data as a 928 training set and the remaining $10 \%$ for testing. Allocation to the training and test set was 929 done randomly and repeated 100 times to obtain a mean and its variance for the achieved 930 decoding accuracy the predictor weights. To identify the classification algorithm which fits 931 our data best we assessed the 25 most used classifiers implemented in MATLAB 932 (Supplementary Fig. 4). Focussing on easy interpretability and high predictive accuracy we 933 chose random subspace ensembles on a linear discriminant analysis (LDA) template 934 (subspace discriminant classifier) for our further analysis, which achieved the highest 935 prediction accuracies compared to all other tested linear classifiers. Briefly, LDA aims to 
936 identify a hyperplane that maximizes the mean distance between the mean of the two

937 classes while minimizing variance between them. Since the sample size of our data was

938 relatively small compared to the number of features, we used the random subspace method,

939 which is a valid approach to resolve this issue and has been shown to be superior to single

940 classification algorithms ${ }^{73,74}$. It operates by creating a classifier ensemble where each

941 classifier is trained with a reduced, randomly sampled number of input features, e.g., they

942 are projected into a new subspace which leads to a relative increase of the number of

943 samples. The number of features to sample in each classifier and the number of learning

944 cycles were set to half of the total number of features and 30 , respectively. The coefficient

945 magnitudes of each feature obtained by each subspace LDA classifier were averaged

946 across learning cycles to get a solid quantification of its predictive value. As measures of

947 classification performance, we used the prediction accuracy, the AUC of the receiver

948 operating characteristic $(\mathrm{ROC})$ and the $\mathrm{F} 1$-score which is defined as follows: $\mathrm{F} 1=$

949 ( $2^{*}$ class1 precision * class1recall / (class2precision + class1recall)). Precision is defined as

950 the True positives / True Positive + False Positive for class 1 and as the True negatives /

951 True Negative + False Negative for class 2. Recall is defined as True Positive / True Positive

952 + False Negative which is equivalent to the sensitivity for class1 and specificity for class2.

953 Since it has been shown that the theoretical chance level of $50 \%$ should not be expected

954 and it is favourable to obtain an empirical chance level, we randomly shuffled the data labels

955 (e.g. correct / incorrect) and repeated the analysis described above to create an empiric null

956 distribution ${ }^{75}$.

\section{Statistical analysis}

958 Behavioural training and challenge data were analysed with repeated-measures ANOVA and 959 pairwise Sidak post-hoc tests for simple main effects. To determine the importance of 960 individual connectivity or activity measures (predictors), we used a two-step procedure: First,

961 pre-classification, we performed a paired $t$-test for each feature comparing its value in 962 correct vs. incorrect trials. Second, post-classification, we used the magnitude of the weight 
963 of each predictor as delivered by the subspace discriminant classifiers to perform a paired $t$ -

964 test between the obtained magnitudes and the magnitudes from the shuffled dataset.

965 Features were only recognized as significantly important if both criteria were met. $P$-values

966 were Bonferroni-corrected within-species for the total number of used features across all

967 classifiers calculated in mice $(1184 ; P<0.05 / 1184)$ and humans $(1584 ; P<0.05 / 1584)$ in

968 the most conservative analysis; additional analysis was conducted to evaluate the

969 dependency of the number of obtained significant predictors with less stringent adjustments.

971 Data and protocol availability

972 All source data for behavioural performance in mice can be obtained from the corresponding

973 author upon reasonable request. All electrophysiological data from mice will be made

974 publicly available at https://gin.g-node.org/KaetzelLab ${ }^{76}$. Human data are available at

975 http://dx.doi.org/10.6080/KOVXODQD. All MATLAB analysis scripts for spectral analysis are

976 publicly available on GitHub (https://github.com/KaetzelLab/LFP_analysis) ${ }^{77}$. Design files of

977 custom-made operant boxes (https://github.com/KaetzelLab/Operant-Box-Design-Files) and

978 task-files for operant WM tasks (https://github.com/KaetzelLab/Operant-Box-Code) are

979 available on GitHub. 


\section{Acknowledgments}

982 We thank Stefanie Schulz for assistance with histology and Dr. Rolf Sprengel (Heidelberg)

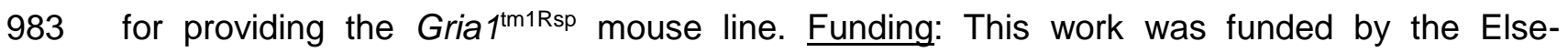

984 Kroener-Fresenius/German-Scholars-Organization Programme for excellent medical

985 scientists from abroad (GSO/EKFS 12; to D.K.), the Juniorprofessorship programme of

986 Baden-Württemberg (to D.K.), the DFG (KA 4594/2-1; to D.K.), the Brain and Behaviour

987 Research Foundation (NARSAD Young Investigator Award 22616 to D.K.), the Alfred-Krupp

988 Foundation (to B.L.), and the National Institute of Neurological Disorders and Stroke

989 (K99NS115918 to E.L.J.).

\section{Author Contributions}

991 SKTK and DS conducted all mouse experiments. DS analysed mouse and human data, with

992 initial support from AMB and BFG. DS and DK designed the study based on prior input from

993 AMB, BFG, and DMB. BL provided essential resources. ELJ provided the human data.

994 SKTK, TA, and DK developed the pyControl operant box enabling simultaneous recording 995 during operant behavioural testing. DS and DK wrote the manuscript with advice from all 996 authors.

\section{Competing Interests Statement}

998 The authors declare no competing interest. 


\section{References}

1. Baddeley, A. D. \& Hitch, G. Working Memory. in Psychology of Learning and Motivation (ed. Bower, G. H.) vol. 8 47-89 (Academic Press, 1974).

2. Baddeley, A. Working memory. Science 255, 556-559 (1992).

3. Millan, M. J. et al. Cognitive dysfunction in psychiatric disorders: characteristics, causes and the quest for improved therapy. Nat. Rev. Drug Discov. 11, 141-168 (2012).

4. Lalonde, R. The neurobiological basis of spontaneous alternation. Neurosci. Biobehav. Rev. 26, 91-104 (2002).

5. D'Esposito, M. et al. The neural basis of the central executive system of working memory. Nature 378, 279-281 (1995).

6. Curtis, C. E., Rao, V. Y. \& D'Esposito, M. Maintenance of spatial and motor codes during oculomotor delayed response tasks. J. Neurosci. 24, 3944-3952 (2004).

7. Goldman-Rakic, P. S. The physiological approach: functional architecture of working memory and disordered cognition in schizophrenia. Biol. Psychiatry 46, 650-661 (1999).

8. Yoon, T., Okada, J., Jung, M. W. \& Kim, J. J. Prefrontal cortex and hippocampus subserve different components of working memory in rats. Learn. Mem. 15, 97-105 (2008).

9. Hallock, H. L., Wang, A. \& Griffin, A. L. Ventral Midline Thalamus Is Critical for Hippocampal-Prefrontal Synchrony and Spatial Working Memory. J. Neurosci. 36, Memory. Curr. Biol. 27, 1829-1835.e4 (2017). lesions disrupt oscillatory signatures of spatiotemporal integration in working memory. Cortex 138, 113-126 (2021). 
1028

1029

1030

1031

1032

1033

1034

1035

1036

1037

1038

1039

1040

1041

1042

1043

1044

1045

1046

1047

1048

1049

1050

1051

1052

1053

1054

13. Johnson, E. L. et al. Spectral Imprints of Working Memory for Everyday Associations in the Frontoparietal Network. Front. Syst. Neurosci. 12, (2019).

14. Parnaudeau, S. et al. Inhibition of Mediodorsal Thalamus Disrupts Thalamofrontal Connectivity and Cognition. Neuron 77, 1151-1162 (2013).

15. Fuster, J. M. \& Alexander, G. E. Neuron Activity Related to Short-Term Memory. Science 173, 652-654 (1971).

16. Cahusac, P. M. B., Miyashita, Y. \& Rolls, E. T. Responses of hippocampal formation neurons in the monkey related to delayed spatial response and object-place memory tasks. Behav. Brain Res. 33, 229-240 (1989).

17. O'Keefe, J. \& Speakman, A. Single unit activity in the rat hippocampus during a spatial memory task. Exp. Brain Res. 68, 1-27 (1987).

18. Watanabe, T. \& Niki, H. Hippocampal unit activity and delayed response in the monkey. Brain Res. 325, 241-254 (1985).

19. Hallock, H. L., Arreola, A. C., Shaw, C. L. \& Griffin, A. L. Dissociable roles of the dorsal striatum and dorsal hippocampus in conditional discrimination and spatial alternation Tmaze tasks. Neurobiol. Learn. Mem. 100, 108-116 (2013).

20. Johnson, E. L. et al. Dynamic frontotemporal systems process space and time in working memory. PLOS Biol. 16, e2004274 (2018).

21. Boran, E. et al. Persistent hippocampal neural firing and hippocampal-cortical coupling predict verbal working memory load. Sci. Adv. 5, eaav3687 (2019).

22. Miller, E. K., Lundqvist, M. \& Bastos, A. M. Working Memory 2.0. Neuron 100, 463-475 (2018).

23. Minzenberg, M. J., Laird, A. R., Thelen, S., Carter, C. S. \& Glahn, D. C. Meta-analysis of 41 functional neuroimaging studies of executive function in schizophrenia. Arch. Gen. Psychiatry 66, 811-822 (2009).

24. Jacob, S. N., Hähnke, D. \& Nieder, A. Structuring of Abstract Working Memory Content by Fronto-parietal Synchrony in Primate Cortex. Neuron 99, 588-597.e5 (2018). 
25. Yamamoto, J., Suh, J., Takeuchi, D. \& Tonegawa, S. Successful Execution of Working Memory Linked to Synchronized High-Frequency Gamma Oscillations. Cell 157, 845857 (2014).

26. Bygrave, A. M. et al. Hippocampal-prefrontal coherence mediates working memory and selective attention at distinct frequency bands and provides a causal link between schizophrenia and its risk gene GRIA1. Transl. Psychiatry 9, 142 (2019).

27. Jones, M. W. \& Wilson, M. A. Theta Rhythms Coordinate Hippocampal-Prefrontal Interactions in a Spatial Memory Task. PLOS Biol. 3, e402 (2005).

28. Duvarci, S. et al. Impaired recruitment of dopamine neurons during working memory in mice with striatal D2 receptor overexpression. Nat. Commun. 9, 2822 (2018).

29. Sigurdsson, T., Stark, K. L., Karayiorgou, M., Gogos, J. A. \& Gordon, J. A. Impaired hippocampal-prefrontal synchrony in a genetic mouse model of schizophrenia. Nature 464, 763-7 (2010).

30. O'Neill, P.-K., Gordon, J. A. \& Sigurdsson, T. Theta Oscillations in the Medial Prefrontal Cortex Are Modulated by Spatial Working Memory and Synchronize with the Hippocampus through Its Ventral Subregion. J. Neurosci. 33, 14211-14224 (2013).

31. Tamura, M., Spellman, T. J., Rosen, A. M., Gogos, J. A. \& Gordon, J. A. Hippocampalprefrontal theta-gamma coupling during performance of a spatial working memory task. Nat. Commun. 8, $2182(2017)$.

32. Tamura, M., Mukai, J., Gordon, J. A. \& Gogos, J. A. Developmental Inhibition of Gsk3 Rescues Behavioral and Neurophysiological Deficits in a Mouse Model of Schizophrenia Predisposition. Neuron 89, 1100-1109 (2016).

33. Spellman, T. et al. Hippocampal-prefrontal input supports spatial encoding in working memory. Nature 522, 309-314 (2015).

34. Maisson, D. J.-N., Gemzik, Z. M. \& Griffin, A. L. Optogenetic suppression of the nucleus reuniens selectively impairs encoding during spatial working memory. Neurobiol. Learn. Mem. 155, 78-85 (2018). 
1082

1083

1084

1085

1086

1087

1088

1089

1090

1091

1092

1093

1094

1095

1096

1097

1098

1099

1100

1101

1102

1103

1104

1105

1106

1107

1108

1109

35. Sanderson, D. J. \& Bannerman, D. M. The role of habituation in hippocampusdependent spatial working memory tasks: Evidence from GluA1 AMPA receptor subunit knockout mice. Hippocampus 22, 981-994 (2012).

36. Cunniff, M. M., Markenscoff-Papadimitriou, E., Ostrowski, J., Rubenstein, J. L. \& Sohal, V. S. Altered hippocampal-prefrontal communication during anxiety-related avoidance in mice deficient for the autism-associated gene Pogz. eLife 9, e54835 (2020).

37. Strahnen, D., Kapanaiah, S. K. T., Bygrave, A. M. \& Kätzel, D. Lack of redundancy between electrophysiological measures of long-range neuronal communication. BMC Biol. 19, 24 (2021).

38. Hultman, R. et al. Brain-wide Electrical Spatiotemporal Dynamics Encode Depression Vulnerability. Cell 173, 166-180.e14 (2018).

39. Hultman, R. et al. Dysregulation of Prefrontal Cortex-Mediated Slow-Evolving Limbic Dynamics Drives Stress-Induced Emotional Pathology. Neuron 91, 439-452 (2016).

40. Kirkby, L. A. et al. An Amygdala-Hippocampus Subnetwork that Encodes Variation in Human Mood. Cell 175, 1688-1700.e14 (2018).

41. Woloszyn, L. \& Sheinberg, D. L. Neural Dynamics in Inferior Temporal Cortex during a Visual Working Memory Task. J. Neurosci. 29, 5494-5507 (2009).

42. Johannesen, J. K., Bi, J., Jiang, R., Kenney, J. G. \& Chen, C.-M. A. Machine learning identification of EEG features predicting working memory performance in schizophrenia and healthy adults. Neuropsychiatr. Electrophysiol. 2, 3 (2016).

43. Combrisson, E. et al. From intentions to actions: Neural oscillations encode motor processes through phase, amplitude and phase-amplitude coupling. Neurolmage 147, 473-487 (2017).

44. Akam, T. et al. pyControl: Open source, Python based, hardware and software for controlling behavioural neuroscience experiments. bioRxiv 2021.02.22.432227 (2021) doi:10.1101/2021.02.22.432227.

45. Teutsch, J. \& Kätzel, D. Operant Assessment of DMTP Spatial Working Memory in Mice. Front. Behav. Neurosci. 13, (2019). 
1110

46. Bolkan, S. S. et al. Thalamic projections sustain prefrontal activity during working memory maintenance. Nat. Neurosci. 20, 987-996 (2017).

47. Brincat, Scott et al. Which Neural Signals are Optimal for Brain-Computer Interface Control? doi:10.3217/978-3-85125-260-6-104.

48. Cuthbert, B. N. \& Insel, T. R. Toward the future of psychiatric diagnosis: the seven pillars of RDoC. BMC Med. 11, 126 (2013).

49. Ma, W. J., Husain, M. \& Bays, P. M. Changing concepts of working memory. Nat. Neurosci. 17, 347-356 (2014).

50. Johnson, E. L., Kam, J. W. Y., Tzovara, A. \& Knight, R. T. Insights into human cognition from intracranial EEG: A review of audition, memory, internal cognition, and causality. J. Neural Eng. 17, 051001 (2020).

51. Majerus, S. et al. Cross-Modal Decoding of Neural Patterns Associated with Working Memory: Evidence for Attention-Based Accounts of Working Memory. Cereb. Cortex N. Y. NY26, 166-179 (2016).

52. Niki, H. \& Watanabe, M. Prefrontal and cingulate unit activity during timing behavior in the monkey. Brain Res. 171, 213-224 (1979).

53. Niki, H. \& Watanabe, M. Cingulate unit activity and delayed response. Brain Res. 110, 381-386 (1976).

54. Gilad, A., Gallero-Salas, Y., Groos, D. \& Helmchen, F. Behavioral Strategy Determines Frontal or Posterior Location of Short-Term Memory in Neocortex. Neuron 99, 814828.e7 (2018).

55. Esmaeili, V. \& Diamond, M. E. Neuronal Correlates of Tactile Working Memory in Prefrontal and Vibrissal Somatosensory Cortex. Cell Rep. 27, 3167-3181.e5 (2019).

56. Insanally, M. N. et al. Spike-timing-dependent ensemble encoding by non-classically responsive cortical neurons. eLife 8, e42409 (2019).

57. Hattori, R., Danskin, B., Babic, Z., Mlynaryk, N. \& Komiyama, T. Area-Specificity and Plasticity of History-Dependent Value Coding During Learning. Cell 177, 1858-1872.e15 (2019). 
58. Zamanillo, D. et al. Importance of AMPA receptors for hippocampal synaptic plasticity but not for spatial learning. Science 284, 1805-11 (1999).

59. Chudasama, Y. \& Robbins, T. W. Dopaminergic Modulation of Visual Attention and Working Memory in the Rodent Prefrontal Cortex. Neuropsychopharmacology 29, 16281636 (2004).

60. Goto, K. \& Ito, I. The asymmetry defect of hippocampal circuitry impairs working memory in ß2-microglobulin deficient mice. Neurobiol. Learn. Mem. 139, 50-55 (2017).

61. Bokil, H., Andrews, P., Kulkarni, J. E., Mehta, S. \& Mitra, P. P. Chronux: A platform for analyzing neural signals. J. Neurosci. Methods 192, 146-151 (2010).

62. Vinck, M., Oostenveld, R., van Wingerden, M., Battaglia, F. \& Pennartz, C. M. A. An improved index of phase-synchronization for electrophysiological data in the presence of volume-conduction, noise and sample-size bias. Neurolmage 55, 1548-1565 (2011).

63. Oostenveld, R., Fries, P., Maris, E. \& Schoffelen, J.-M. FieldTrip: Open Source Software for Advanced Analysis of MEG, EEG, and Invasive Electrophysiological Data. Comput. Intell. Neurosci. 2011, (2010).

64. Dhamala, M., Rangarajan, G. \& Ding, M. Analyzing information flow in brain networks with nonparametric Granger causality. Neurolmage 41, 354-362 (2008).

65. Baccalá, L. A. \& Sameshima, K. Partial directed coherence: a new concept in neural structure determination. Biol. Cybern. 84, 463-474 (2001).

66. Dhamala, M., Rangarajan, G. \& Ding, M. Estimating Granger Causality from Fourier and Wavelet Transforms of Time Series Data. Phys. Rev. Lett. 100, 018701 (2008).

67. Dhamala, M., Liang, H., Bressler, S. L. \& Ding, M. Granger-Geweke causality: Estimation and interpretation. Neurolmage 175, 460-463 (2018).

68. Canolty, R. T. \& Knight, R. T. The functional role of cross-frequency coupling. Trends Cogn. Sci. 14, 506-515 (2010).

69. Sirota, A. et al. Entrainment of Neocortical Neurons and Gamma Oscillations by the Hippocampal Theta Rhythm. 60, 683-697 (2008). 
1165

1166

1167

1168

1169

1170

1171

1172

1173

1174

1175

1176

1177

1178

1179

1180

1181

1182

1183

1184

70. Tort, A. B. L. et al. Dynamic cross-frequency couplings of local field potential oscillations in rat striatum and hippocampus during performance of a T-maze task. Proc. Natl. Acad. Sci. 105, 20517-20522 (2008).

71. Voytek, B. et al. Shifts in Gamma Phase-Amplitude Coupling Frequency from Theta to Alpha Over Posterior Cortex During Visual Tasks. Front. Hum. Neurosci. 4, (2010).

72. Chawla, N. V., Bowyer, K. W., Hall, L. O. \& Kegelmeyer, W. P. SMOTE: synthetic minority over-sampling technique. J. Artif. Intell. Res. 16, 321-357 (2002).

73. Skurichina, M. \& Duin, R. P. W. Bagging, Boosting and the Random Subspace Method for Linear Classifiers. Pattern Anal. Appl. 5, 121-135 (2002).

74. Tin Kam Ho. The random subspace method for constructing decision forests. IEEE Trans. Pattern Anal. Mach. Intell. 20, 832-844 (1998).

75. Combrisson, E. \& Jerbi, K. Exceeding chance level by chance: The caveat of theoretical chance levels in brain signal classification and statistical assessment of decoding accuracy. J. Neurosci. Methods 250, 126-136 (2015).

76. Strahnen, D., Kapanaiah, S., Bygrave, A. M. \& Kätzel, D. KaetzelLab/GluA1KO_LFP_data. Ger. Neuroinformatics Node (2020) doi:10.12751/g-node.3lvs3q.

77. Strahnen, D. \& Kätzel, D. KaetzelLab/LFP_analysis. Github (2020) doi:10.5281/zenodo.4384980. 